\title{
HOT GAS IN THE COLD DARK MATTER SCENARIO: X-RAY CLUSTERS FROM A HIGH-RESOLUTION NUMERICAL SIMULATION
}

\author{
Hyesung Kang, Renyue Cen, Jeremiah P. Ostriker, and Dongsu Ryu \\ Princeton University Observatory, Princeton, NJ 08544 \\ Received 1993 June 28 ; accepted 1993 December 9
}

\begin{abstract}
A new, three-dimensional, shock-capturing hydrodynamic code is utilized to determine the distribution of hot gas in a standard cold dark matter (CDM) model of the universe. Periodic boundary conditions are assumed: a box with size $85 h^{-1} \mathrm{Mpc}$ having cell size $0.31 h^{-1} \mathrm{Mpc}$ is followed in a simulation with $270^{3}=10^{7.3}$ cells. Adopting standard parameters determined from $C O B E$ and light-element nucleosynthesis, $\sigma_{8}=1.05, \Omega_{b}=0.06$, and assuming $h=0.5$, we find the X-ray-emitting clusters and compute the luminosity function at several wavelengths, the temperature distribution, and estimated sizes, as well as the evolution of these quantities with redshift. We find that most $\left(\geq \frac{3}{4}\right)$ of the total $\mathrm{X}$-ray $(h v>0.3 \mathrm{keV})$ emissivity in our box originates in a relatively small number of identifiable clusters which occupy approximately $10^{-3}$ of the box volume. This standard CDM model, normalized to $C O B E$, produces approximately 5 times too much emission from clusters having $L_{x}>10^{43}$ ergs s${ }^{-1}$, a not-unexpected result. If all other parameters were unchanged, we would expect adequate agreement for $\sigma_{8}=0.6$. This provides a new and independent argument for lower small-scale power than standard CDM at the $8 h^{-1} \mathrm{Mpc}$ scale. The background radiation field at $1 \mathrm{keV}$ due to clusters in this model is approximately one-third of the observed background, which, after correction for numerical effects, again indicates approximately 5 times too much emission and the appropriateness of $\sigma_{8}=$ 0.6. If we have used the observed ratio of gas to total mass in clusters, rather than basing the mean density on light-element nucleosynthesis, then the computed luminosity of each cluster would have increased still further, by a factor of approximately 10 .

The number density of clusters increases to $z \sim 1$, but the luminosity per typical cluster decreases, with the result that evolution in the number density of bright clusters is moderate in this redshift range, showing a broad peak near $z=0.7$, and then a rapid decline above redshift $z=3$. Detailed computations of the luminosity functions in the range $L_{x}=10^{40}-10^{44} \mathrm{ergs} \mathrm{s}^{-1}$ in various energy bands are presented for both cluster central regions $\left(r \leq 0.5 h^{-1} \mathrm{Mpc}\right)$ and total luminosities $\left(r<1 h^{-1} \mathrm{Mpc}\right)$ to be used in comparison with ROSAT and other observational data sets. The quantitative results found disagree significantly with those found by other investigators using semianalytic techniques. For example, the total volume emission from hot cluster gas is found to increase by about a factor of 1.5 between $z=0$ and $z=1$, but for the same CDM model Kaiser (1986) predicted an increase of a factor of 5.7, for self-similar evolution of clusters.

We find little dependence of core radius on cluster luminosity and a dependence of temperature on luminosity given by $\log k T_{x}=A+B \log L_{x}$, which is slightly steeper $(B=0.38)$ than is indicated by observations. Computed temperatures are somewhat higher than observed, as expected, in that $C O B E$-normalized CDM has too much power on the relevant scales. A modest average temperature gradient is found, with temperatures dropping to $90 \%$ of central values at $0.4 h^{-1} \mathrm{Mpc}$ and $70 \%$ of central values at $0.9 h^{-1} \mathrm{Mpc}$. In these models the decrease of the core radius and temperature with redshift is significant (in rough accord with the analytic calculations). We do not expect to see the same result in open-universe models, so this property should provide an important discriminant among cosmological models.

Examining the ratio of gas to total mass in the clusters (which we find to be antibiased by a factor of approximately 0.6 ), normalized to $\Omega_{b} h^{2}=0.015$, and comparing with observations, we conclude, in agreement with White (1991), that the cluster observations argue for an open universe.

Subject headings: cosmology: theory - galaxies: clustering — hydrodynamics large-scale structure of universe - X-rays : galaxies
\end{abstract}

\section{INTRODUCTION}

X-ray emission from clusters of galaxies provides a powerful cosmological probe. When first discovered (Gursky et al. 1971), the prodigous luminosities $\left(\sim 10^{44} \mathrm{ergs}^{-1}\right)$ were a cause of some surprise, but in retrospect it is clear that we should have expected such objects to exist. Zwicky (1933) had firmly established the existence of these assemblies with masses of order $10^{15} M_{\odot}$ and gravitational radii of order $1 \mathrm{Mpc}$. These numbers, and an assumed gas/total mass of order $10 \%$, lead directly to the prediction of luminosity in the $1-10 \mathrm{keV}$ range in excess of $10^{43} \mathrm{ergs} \mathrm{s}^{-1}$.
But theoretical considerations alone drive one to expect that this type of object should exist, with the cataloged "clusters" being only the extreme and easily identifiable members of the class. The growth of perturbations in any variant of the many theories for the origin of structure leads to the "breaking" of nonlinear waves, in a fashion not altogether unlike the breaking of ocean waves or the steepening of sound waves into shocks. Then, allowing for the gas content of the universe, at the surfaces where these caustics occur (cf. Zel'dovich 1970), real gaseous shocks will form with post shock temperatures of order $\left(k T / m_{0}\right)=(3 / 256)\left(H_{0} \lambda\right)^{2}$. Intersections of sheets result in 
still hotter and denser filaments, and intersections of filaments with sheets produce clumps, vertices, where the density, gravitational potential, and temperature are maximal. These regions also collect galaxies resulting in optically identifiable "clusters of galaxies." Thus the existence of "X-ray clusters" could have been anticipated.

As observations of X-ray clusters have improved (cf. Bahcall 1993 for a recent review), comparison of the output of theoretical models for the origin of structure with the rapidly improving real data has become imperative. But the problem is computationally difficult. Given that the core radius of the typical cluster is less than $0.5 h^{-1} \mathrm{Mpc}$ and the cluster-cluster separation of order $50 h^{-1} \mathrm{Mpc}$, a resolution of $100^{3}=10^{6}$ cells is the absolute minimum required to compute any statistically valid quantities. This has been beyond the capacity of our technology until fairly recently. Kaiser (1986), in a classic paper on this subject, says, "We [would] need full three dimensional hydrodynamical simulations which are not at present a viable proposition."

Thus, other simpler, indirect methods were utilized. In addition to the first-order analytic estimates of mean quantities (Cole \& Kaiser 1989; Cavaliere, Menci, \& Setti 1991), another type of approach, which is essentially static but can simulate very large volumes, has been followed. To address this problem in an ingeniously economical if approximate way, Bond \& Myers (1991a, b) modeled all nonlinear structure using the Press-Schechter formalism for the growth of dark matter clusters and then added to each simulated cluster (hot) gas in virial equilibrium. This approach uses idealized clusters, which are laid down in huge volumes according to the general prescriptions of a cosmological scenario, through mass functions based on the number density and the distribution of peaks of the density fluctuations (Kaiser 1986; Bond 1990; Bond \& Myers 1991a, b; Blanchard et al. 1992), or to the observed cluster abundances and characteristics, which then are extrapolated backward in time (Markevitch et al. 1991). Then the projected sky distribution is derived, and from it the values of observables of interest. Recent applications of the Press-Schechter formalism by Evrard \& Henry (1991) and Blanchard et al. (1992) have aimed at predicting the ROSAT cluster observations. Another approach is to adopt "constrained initial conditions" (for recent applications of this method see Evrard, Summers, \& Davis 1994). This enables one to use a small volume which still contains a cluster. Resolution is gained, but statistical information is lost.

We have been following a more "brute force" approach with increasingly accurate numerical methods. In Cen et al. (1990) we used the Jameson (1989) code with cell number $N=100^{3}$ in a box of size $L=30 h^{-1} \mathrm{Mpc}$ to model the X-ray luminosity function in a cold dark matter (CDM) scenario. This was improved on in a $128^{3}\left(L=64 h^{-1} \mathrm{Mpc}\right)$ simulation using the same code (Cen \& Ostriker 1992) and still further with a $N=200^{3}\left(L=80 h^{-1} \mathrm{Mpc}\right)$ run (Cen \& Ostriker 1993).

In all of these computations the resolution $\Delta l$ was somewhat worse than $2.5\left(L / N^{1 / 3}\right) h^{-1} \mathrm{Mpc}$, since the diffusive Jameson code spreads a shock over several cells. In the three quoted calculations the cell size was approximately $0.4 h^{-1} \mathrm{Mpc}$, which thus corresponded to an effective top-hat resolution of radius $\Delta r>0.4 \times 2.5(3 / 4 \pi)^{1 / 3}=0.62 h^{-1} \mathrm{Mpc}$, or a Gaussian of $r_{g}>0.36 h^{-1} \mathrm{Mpc}$. Real clusters often have core radii which are smaller by almost a factor of 2 than this scale, so these calculations suffered significantly from a lack of resolution. In the earlier papers we attempted to compensate for this error using extrapolation procedures detailed in Cen (1992). But of course it is better to simply increase the numerical resolution. Recently, we have developed a shock-capturing "total variation diminishing" (TVD) code (Ryu et al. 1993), with considerably improved resolution, which enables us to do this. In estimating resolution, the ability to treat a shock (tests shown in Ryu et al. 1993) is just one factor. The ability to handle continuous flow, the amount of unwanted numerical viscosity, etc., must also be considered. A detailed description of the new method, its derivation and origin, and the tests of its accuracy are reported on elsewhere (Ryu et al. 1993). It suffices to note here that we expect that the resolution of the new code (for a factor of 4 increase in a shock) is $2-3$ cells and superior by a factor of 2-3 to our previous work.

Here we combine this superior code with a larger scale run, $N=270^{3}=10^{7.3}$, using a slightly larger box, $L=85 h^{-1} \mathrm{Mpc}$, and the new COBE (Smoot et al. 1992) normalization of the CDM spectrum to produce a reasonably accurate computation of the X-ray-emitting properties of the CDM model. An estimate of the numerical reliabilty of our results may be obtained by comparing the results of this paper with those of the paper by Bryan et al. (1994a), which utilizes a totally independent computational scheme but identical initial conditions and identical physical modeling assumptions. The very brightest clusters are intrinsically rare objects. This has led us to take larger and larger volumes in successive simulations in order to be better able to represent the bright end of the luminosity function. We suspect that we have not yet reached a sufficiently large box in the present run and that our high-luminosity cutoff may be affected by this bias. Section 2 describes the method and initial conditions, $\S 3$ the results, with a comparison with observations and discussions in $\S 4$.

\section{METHOD AND INITIAL CONDITIONS}

\subsection{Method}

The superiority of the new TVD code to our previous code (cf. Cen 1992) can be resolved into three aspects: (1) The TVD code is able to capture a shock in 2-3 cells rather than 4-7 cells. The method (Harten 1983) has elements in common with the piecewise parabolic method (PPM) code developed by Norman and colleagues (Colella \& Woodward 1984; Bryan et al. 1994b). (2) A new variable (entropy $S$ ) is added to the code, which enables us to handle very strong (high Mach number) shocks without generating artificial, nonphysical heating of the gas in regions outside the shocks. Such spurious heating is unavoidable in conventional codes, where only the total energy is used as a variable and pressure is obtained by subtracting the kinetic energy from the total energy. In most codes a slight error in the calculation of the total energy and/or kinetic energy results in a large error in computing the pressure (or temperature). (3) A main difference between cosmological applications and normal laboratory hydrodynamics is the inclusion of the self-gravitational forces. It is unavoidable that density diffusion (an inevitable error in all numerical codes) in a gravitational field does some work, which affects the momentum and energy calculation. We take special care in handling this problem in such a way that energy conservation (represented by the Layzer-Irvine equation) of the code is not broken and the inevitable errors are primarily in the large terms (kinetic and gravitational energy), not in the relatively smaller thermal energy.

We have performed various tests on the code. A standard 
shock-tube test shows that the code resolves the shock in 2-3 cells. A one-dimensional cosmological pancake collapse calculation indicates that the code conserves energy at $(1 \%, 0.01 \%)$ for $(32,1024)$ cells. A three-dimensional calculation of the standard CDM model with $128^{3}$ cells, $L=64 h^{-1} \mathrm{Mpc}$, and $\sigma_{8}=1$ conserves the energy to $4 \%$. The detailed presentation of the code with various test results has been given in Ryu et al. (1993).

The simulation reported on in this paper did not include any atomic processes, i.e., no cooling or heating was added, except for the adiabatic cooling due to the general expansion of the universe, and "heating" occurs only due to adiabatic compression or to entropy generation at shock fronts. For the hot gas, which we will discuss in this paper, this approximation is valid, since the cooling time exceeds the Hubble time by a fair margin. A Courant number of 0.95 was used, which we found to be appropriate for this calculation after various tests.

\subsection{Initial Conditions}

We adopt the standard CDM power spectrum with the transfer function given by Bardeen et al. (1986). The following parameters are used: $n=1, h=0.5, \Omega=1, \Omega_{b}=0.06$, and $\sigma_{8}=1.05$. Note that the amplitude normalization of the power spectrum is determined by $C O B E$ observation (cf. Efstathiou, Bond, \& White 1992), parameterized by $\sigma_{8}$ to translate into conventional notation. Our box size is $85 h^{-1} \mathrm{Mpc}$ with $270^{3}$ cells and $135^{3}$ dark matter particles, so our nominal resolution is $0.31 h^{-1} \mathrm{Mpc}$ with our real spatial resolution a factor of 2-3 worse than this. The choice of $\Omega_{b}$ is consistent with lightelement nucleosynthesis (Walker et al. 1991).

\section{RESULTS}

The X-ray clusters in the simulation are identified as follows. We first calculate the total X-ray luminosity due to thermal bremsstrahlung:

$$
L_{\mathrm{ff}}=4 \pi \int_{v} \int_{V} j_{\mathrm{ff}} d^{3} r d v
$$

for each cell given the cell density and temperature, where $j_{\mathrm{ff}}$ is given by (in units of ergs $\mathrm{cm}^{-3} \mathrm{~s}^{-1} \mathrm{~Hz}^{-1} \mathrm{sr}^{-1}$ )

$$
\begin{aligned}
j_{\mathrm{ff}}(v)= & \frac{1}{4 \pi} \frac{32 e^{4} h}{3 m_{e}^{2} c^{3}}\left[\frac{\pi h v_{0}(\mathrm{H})}{3 k T}\right]^{1 / 2} e^{-h v / k T} \\
& \times g_{\mathrm{ff}}(T, v)[n(\mathrm{H} \mathrm{II})+n(\mathrm{He} \mathrm{II})+4 n(\mathrm{He} \mathrm{III})] n(e),
\end{aligned}
$$

which assumes cosmic abundances with both hydrogen and helium fully ionized, and $g_{\mathrm{ff}}(T, v)$ is the Gaunt factor (Spitzer 1978). No allowance is made for line emission, which will be treated in a subsequent paper.

The cells with the total X-ray luminosity higher than $10^{38}$ ergs $s^{-1}$ are selected as X-ray-bright cells. Then we find the local maxima (by comparing $L_{\mathrm{ff}}$ of each X-ray-bright cell with that of 26 neighboring cells) and identify them as the centers of the X-ray clusters. Having defined the centers of the X-ray clusters, we go back to the whole simulation box to define these clusters. We analyze the simulation in two different ways (which correspond to spheres of radius 0.5 and $1.0 h^{-1} \mathrm{Mpc}$ ) as follows: First, each cluster core consists of 27 cells $(26$ cells surrounding the central cell, plus the central cell). These 27 cells are weighted so that the total volume of the cluster equals the volume of a sphere of radius $0.5 h^{-1} \mathrm{Mpc}$, as appropriate for observationally defined X-ray clusters. That is, we define a weight $w \equiv R_{v}$ for the central cell and the 6 cells that share the faces with the central cell, $w \equiv \frac{3}{4} R_{v}$ for 12 cells that share the edges with the central cell, and $w \equiv \frac{1}{2} R_{v}$ for 8 cells that share only the corners with the central cell, where $R_{v}=(4 \pi$ $\left.\times 0.5^{3}\right) /\left(20 \Delta x^{3}\right)=0.839$. This weighting scheme compensates for the adoption of slightly too large a volume by lowering the weight per cell. For concentrated clusters we may be underestimating the luminosity by as much as $16 \%$, a correction small compared with other errors. The "core" luminosities calculated here are from regions $r h<0.5 \mathrm{Mpc}$, but the true X-ray cores as defined by equation (4) are about a factor of 2 smaller. Second, the total X-ray cluster volume consists of 125 cells (124 cells surrounding the central cell, plus the central cell), and each cell is weighted equally, which gives a volume equivalent to a sphere of radius $0.98 h^{-1} \mathrm{Mpc}$.

Our $\left(85 h^{-1} \mathrm{Mpc}\right)^{3}$ box at $z=0$ contained $(0,0)$ clusters with (total, core) luminosity brighter than $10^{45}$ ergs $\mathrm{s}^{-1},(8,1)$ brighter than $10^{44}$ ergs s ${ }^{-1},(29,17)$ brighter than $10^{43}$ ergs $\mathrm{s}^{-1},(115,68)$ brighter than $10^{42}$ ergs $^{-1}$, and $(367,264)$ brighter than $10^{41}$ ergs $^{-1}$. We attribute the lack of clusters brighter

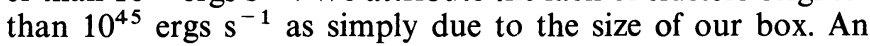
additional factor of at least 2 in scale (and 8 in computer resources) would be required to improve significantly on the quoted results.

It is convenient to fit the luminosity function to the threeparameter Schechter function

$$
n(L) d L=n_{0}\left(L / L_{*}\right)^{-\alpha} e^{-L / L^{*}} d\left(L / L^{*}\right) .
$$

The raw results for the luminosity functions are shown in Figures $1 a, 2 a, 3 a$, and $4 a$ respectively for total luminosity and for $0.3-3.5 \mathrm{keV}, 0.5-4.5 \mathrm{keV}$, and $2-10 \mathrm{keV}$ bands emitted from central $0.5 h^{-1} \mathrm{Mpc}$ regions (core) of each cluster, and in Figures $1 b, 2 b, 3 b, 4 b$ and for $1 h^{-1} \mathrm{Mpc}$ spheres. The figures show the range of cluster properties which are most accessible to observation (in the range we are able to compute) $10^{40} \mathrm{ergs}$ $\mathrm{s}^{-1} \leq L_{x} \leq 10^{44} \mathrm{ergs} \mathrm{s}^{-1}$ and $0 \leq z \leq 1$. We have computed approximate Schechter function $\left[n(L) d L \equiv n_{0}(z)\left(L / L^{*}\right)^{-\alpha}\right.$ $\left.\exp \left(-L / L^{*}\right) d L\right]$ fits to the results with the numerical parameters $\left(n_{0}, L^{*}, \alpha\right)$ as a function of redshift collected in Table 1 and the data extended to $z=5$. Luminosities and temperatures are presented as they would be seen by observers near the clusters, but densities and emissivities are in comoving coordinates. Also, in Table 1 we integrated over the cluster luminosity function, using the Schechter fit $j_{\mathrm{cl}} \equiv n_{0} L^{*} \Gamma(2-\alpha)$ (in units of $10^{40}$ ergs $\mathrm{s}^{-1} h^{-3}$ comoving $\mathrm{Mpc}^{3}$ ), showing the result in the second to last column, and give also in the last column the total emissivity from the box as $j_{\text {gas }}$, which includes the emission from lower regions farther from cluster cores than $0.5 h^{-1} \mathrm{Mpc}$ and also from clusters whose central emissivity is less than our cutoff value.

We see that the cluster cores (upper panels of Table 1), as we have defined them, contain between one-half and one-fourth of the total X-ray emission in the regions studied. The total cluster luminosity (lower panels of Table 1) in the box is typically three-fourths of the X-ray emission from the box, although the bright clusters $\left(L \geq 10^{43}\right.$ ergs $\left.\mathrm{s}^{-1}\right)$ occupy a volume which is less than $10^{-3}$ of the total, i.e., the emissivity is very concentrated at bright peaks. For the total luminosity, the Schechter $\alpha$-parameter is approximately 1.5 with little evolution, and $\alpha$ for the few $\mathrm{keV}$ bands is typically slightly flatter at 1.4. For $\alpha<2$, as we have noted, most of the luminosity arises from bright clusters.

We defer a detailed comparison with observations to a subsequent paper. For the present we compare in Figure $1 b$ the 


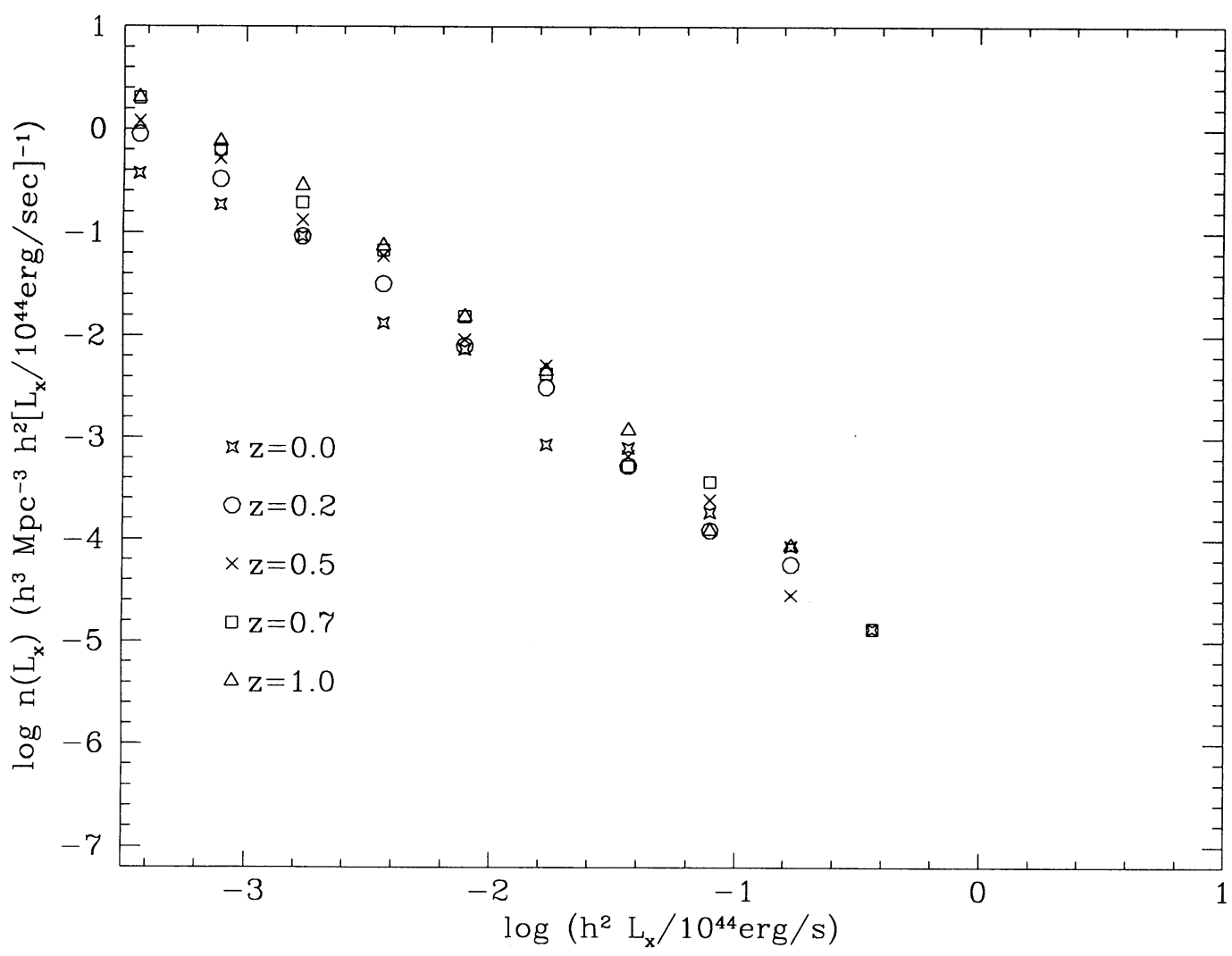

Fig. $1 a$

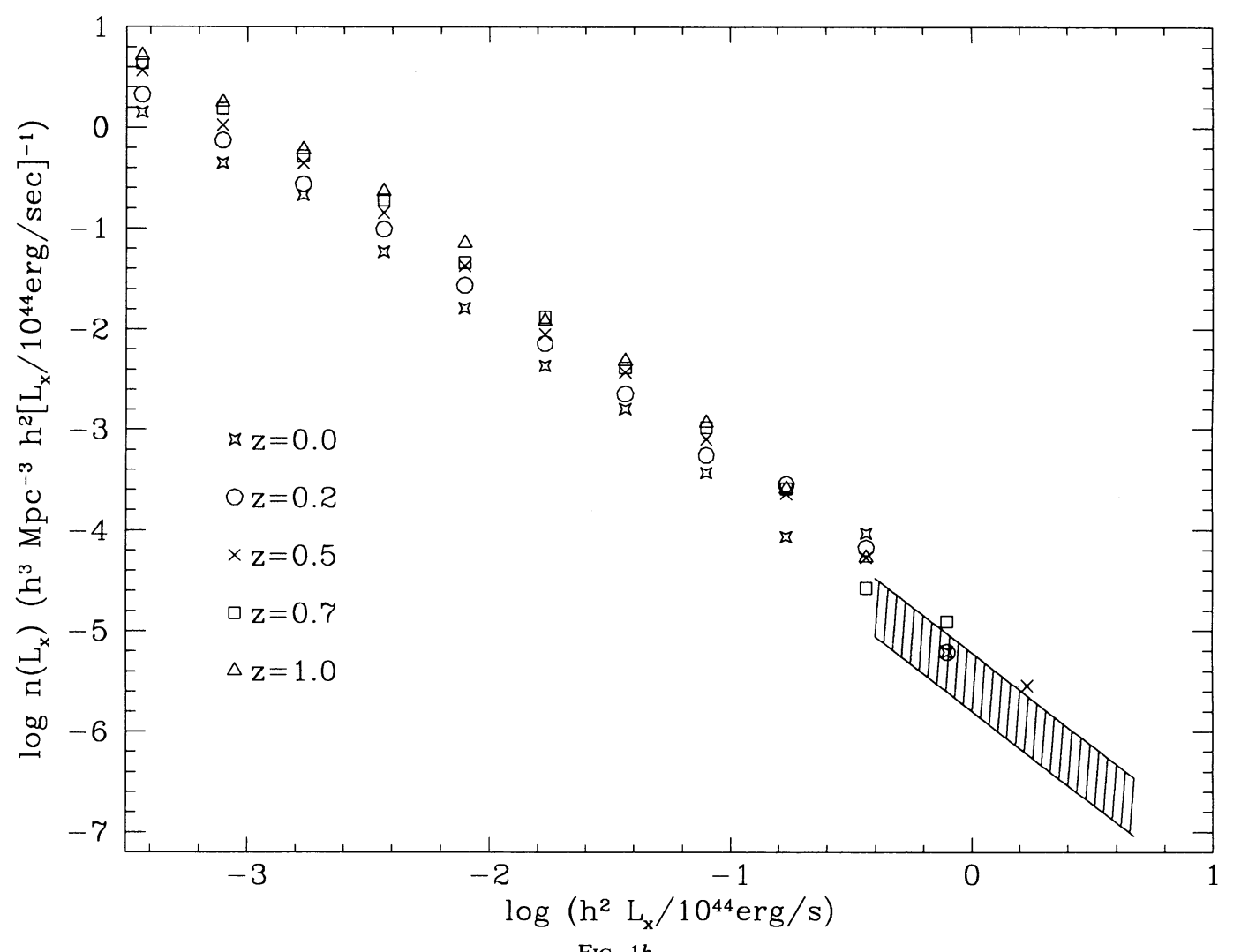

Fig. 1.-(a) X-ray cluster bremsstrahlung luminosity function (from central $<0.5 h^{-1} \mathrm{Mpc}$ regions) integrated over the whole frequency range at five different redshifts, $z=(0,0.2,0.5,0.7,1.0)$. (b) X-ray cluster bremsstrahlung luminosity function (from $<1.0 h^{-1} \mathrm{Mpc}$ region) integrated over the whole frequency range at the same five redshifts as in $(a)$. The absence of line emission from computed luminosities leads to an underestimate of the bolometric luminosity. The hatched area shows the observations (Henry \& Arnaud 1991:

$$
\left.\left\{3.1_{-1.8}^{+4.5} \times 10^{-6} h^{3} \mathrm{Mpc}^{-3} h^{2}\left[L_{44}(\mathrm{bol})\right]^{-1}\right\}\left[h^{2} L_{44}(\mathrm{bol})\right]^{-1.85 \pm 0.4}\right) .
$$

Notice that there is minimal evolution (for comoving observers) in contrast to the expectation (Kaiser 1986) of $(1+z)^{5 / 2}$ for self-similar evolution. 

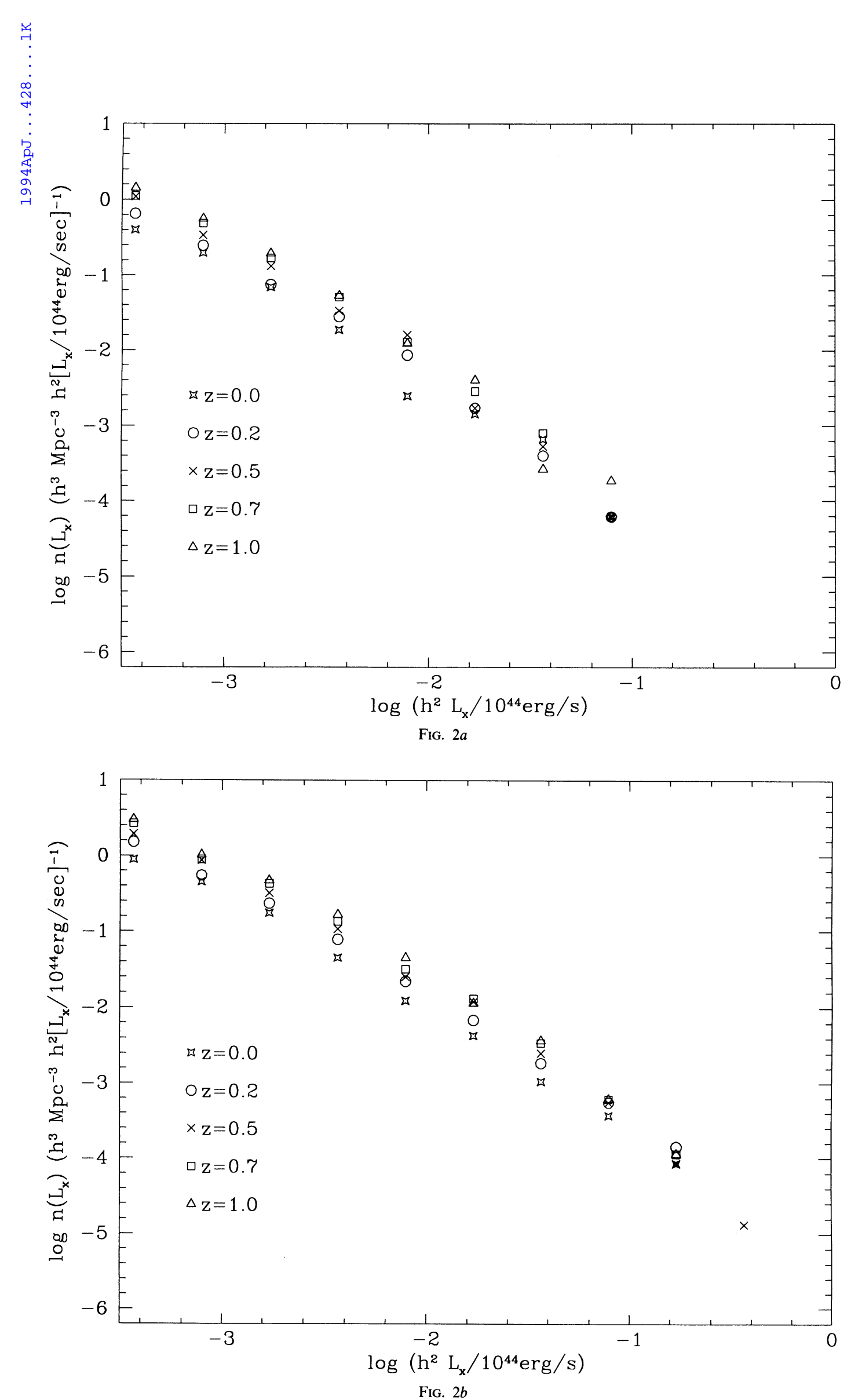

Fig. 2.-Same as Fig. 1, but for the luminosities integrated over the $0.3-3.5 \mathrm{keV}$ frequency band 


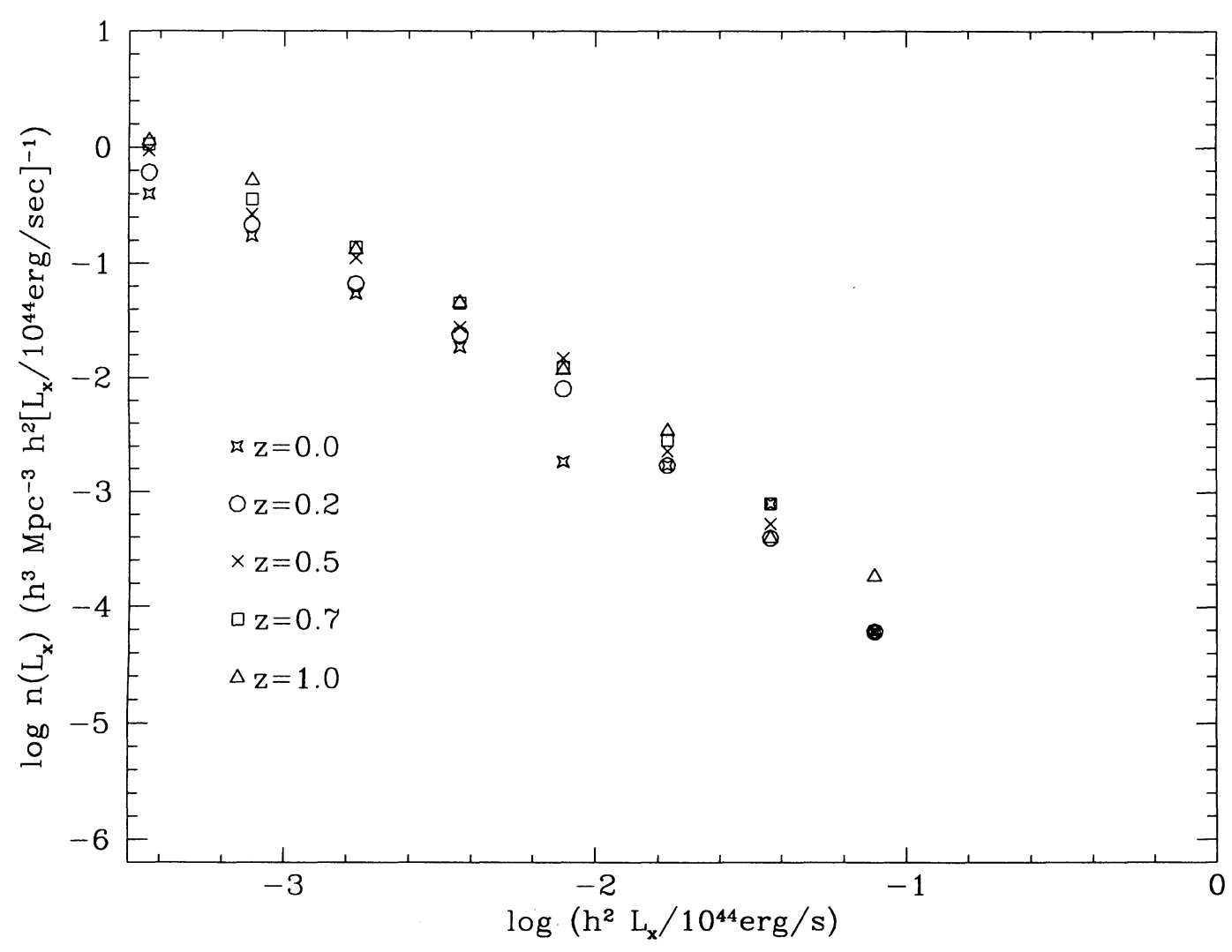

Fig. $3 a$

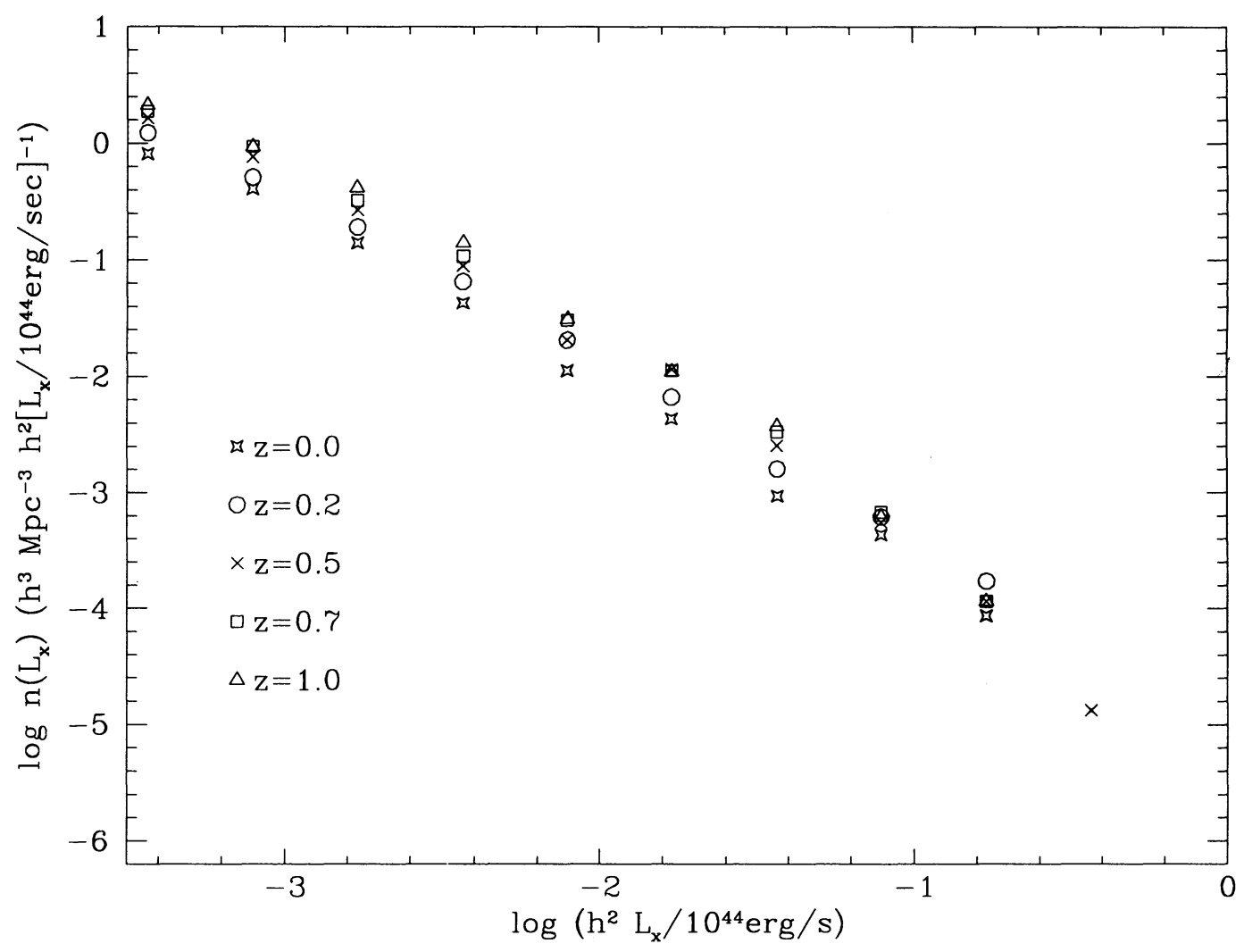

FIG. $3 b$

Fig. 3. - Same as Fig. 1, but for the luminosities integrated over the $0.5-4.5 \mathrm{keV}$ frequency band. Line emission is not significant in this energy range. 

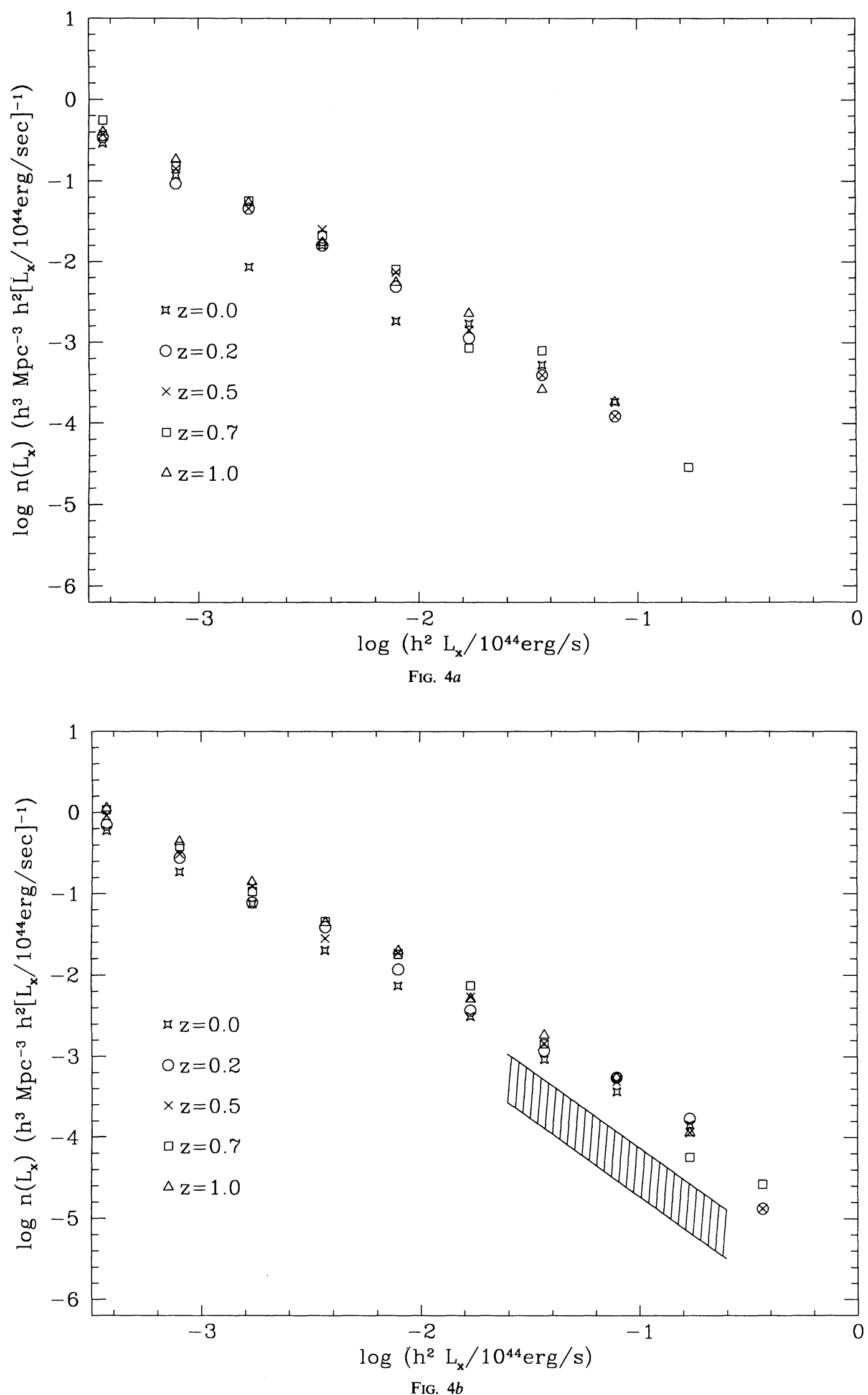

Fig. 4. - Same as Fig. 1, but for the luminosities integrated over the $2-10 \mathrm{keV}$ frequency band. The hatched area shows observational data taken from the review by Henry (1992). The excess of computed over observed is significant and would be considerably greater if we had a numerically more accurate calculation and/or used the observed rather than the computed gas density in clusters. 
TABLE $1 \mathrm{~A}$

PARAMETERS OF SCHEChTER FITS FOR THE X-RAY Cluster LuMINOSITY FunCTION INTEGRATED OVER THE ENTIRE FREQUENCY RANGE

\begin{tabular}{|c|c|c|c|c|c|c|c|c|}
\hline $\begin{array}{l}z \\
(1)\end{array}$ & $\begin{array}{l}\alpha \\
(2)\end{array}$ & $\begin{array}{l}L_{x}^{*} \\
(3)\end{array}$ & $\begin{array}{l}k \bar{T}_{x} \\
(4)\end{array}$ & $\begin{array}{l}n_{0} \\
(5)\end{array}$ & $\begin{array}{l}n\left(L>10^{43}\right) \\
(6)\end{array}$ & $\begin{array}{l}n\left(L>10^{44}\right) \\
(7)\end{array}$ & $\begin{array}{l}j_{\mathrm{cl}} \\
(8)\end{array}$ & $\begin{array}{l}j_{\text {gas }} \\
\text { (9) }\end{array}$ \\
\hline \multicolumn{9}{|c|}{ X-Ray Cluster Core Luminosity $\left(<0.5 h^{-1} \mathrm{Mpc}\right)$} \\
\hline $0 \ldots$ & 1.47 & 1.49 & 8.55 & 4.85 & 9.39 & 0.79 & 0.12 & 0.43 \\
\hline 0.2 . & 1.47 & 1.02 & 4.88 & 9.36 & 13.5 & 0.76 & 0.16 & 0.52 \\
\hline ............ & 1.47 & 0.52 & 5.20 & 20.1 & 10.8 & 0.39 & 0.18 & 0.62 \\
\hline $0.7 \ldots \ldots \ldots \ldots$ & 1.51 & 0.68 & 3.14 & 18.0 & 19.1 & 0.57 & 0.22 & 0.62 \\
\hline $1 \ldots \ldots \ldots \ldots \ldots$ & 1.48 & 0.46 & 2.90 & 30.1 & 21.2 & 0.31 & 0.24 & 0.62 \\
\hline & 1.59 & 0.46 & 1.71 & 19.2 & 14.8 & 0.17 & 0.19 & 0.48 \\
\hline $3 \ldots \ldots \ldots \ldots \ldots$ & 1.63 & 0.24 & 0.71 & 34.1 & 11.8 & 0.017 & 0.20 & 0.45 \\
\hline $5 \ldots \ldots \ldots \ldots \ldots$ & 1.97 & 0.16 & 0.20 & 5.39 & 0.99 & $9.6 \times 10^{-5}$ & 0.28 & 0.17 \\
\hline \multicolumn{9}{|c|}{ X-Ray Cluster Total Luminosity $\left(<1 h^{-1} \mathrm{Mpc}\right)$} \\
\hline $0 \ldots$ & 1.44 & 3.33 & 6.08 & 5.23 & 16.5 & 2.53 & 0.28 & 0.43 \\
\hline $0.2 \ldots \ldots \ldots \ldots \ldots$ & 1.43 & 1.89 & 3.98 & 12.1 & 25.9 & 2.84 & 0.36 & 0.52 \\
\hline $0.5 \ldots \ldots \ldots \ldots \ldots$ & 1.44 & 1.30 & 3.56 & 18.9 & 31.5 & 2.45 & 0.39 & 0.62 \\
\hline $0.7 \ldots \ldots \ldots \ldots$ & 1.48 & 1.88 & 2.51 & 14.8 & 34.6 & 3.46 & 0.47 & 0.62 \\
\hline $1 \ldots \ldots \ldots \ldots \ldots$ & 1.44 & 0.93 & 1.98 & 33.0 & 42.5 & 2.26 & 0.49 & 0.62 \\
\hline $2 \ldots \ldots \ldots \ldots \ldots$ & 1.53 & 0.56 & 1.15 & 32.5 & 29.2 & 0.59 & 0.34 & 0.48 \\
\hline $3 \ldots \ldots \ldots \ldots \ldots$ & 1.53 & 0.26 & 0.54 & 67.5 & 25.1 & 0.060 & 0.33 & 0.45 \\
\hline $5 \ldots \ldots \ldots \ldots \ldots$ & 1.91 & 0.20 & 0.17 & 9.62 & 2.68 & $9.8 \times 10^{-4}$ & 0.20 & 0.17 \\
\hline
\end{tabular}

NoTE.-Here $L_{x}^{*}$ is in units of $10^{44} \mathrm{ergs} \mathrm{s}^{-1}, k \bar{T}$ is in $\mathrm{keV}, n_{0}, n\left(L>10^{43}\right)$, and $n\left(L>10^{44}\right)$ are in units of $10^{-6} h^{3} \mathrm{Mpc}^{-3} ; j_{\mathrm{cl}}$ and $j_{\text {gas }}$ are in units of $10^{40} \mathrm{ergs} \mathrm{s}^{-1} h^{-3} \mathrm{Mpc}^{-3}$, and $j_{\mathrm{cl}}$ may be larger than $j_{\mathrm{gas}}$ due to the inaccuracy of the Schechter fit.

bolometric luminosity distribution with that given by Henry \& Arnaud (1991), and in Figure $4 b$ the $2-10 \mathrm{keV}$ luminosity function of low-redshift clusters, as summarized by Henry (1992, Fig. 1), with our computational results. The observational error is approximately a factor of 2 , but the difference between computed and observed luminosity functions in Figure $4 b$ is approximately a factor of 6 (at $L_{x}=10^{43.5} \mathrm{ergs} \mathrm{s}^{-1}$ ) and is probably significant, especially as our numerical procedures tend to systematically underestimate the predicted luminosity. Taking this underestimate to be a factor of 1.8 (cf. Cen 1992 for the procedure), the discrepancy corresponds (taking $j_{v} \propto \sigma_{8}^{4.3}$ ) to a factor of 1.7 in amplitude. Another way to check the consistency of this scaling scheme is as follows: A model with $\sigma_{8}=0.6-0.7$ corresponds approximately to $z=0.5$ for a $\sigma_{8}=$ 1.05 model. Since $n_{e}$ goes as $(1+z)^{3}$ and temperature as $(1+z)^{-1}$, the total luminosity of each cluster will go as $n_{e}^{2} T^{1 / 2} \propto(1+z)^{11 / 2}=9.3$ (if the physical size of a cluster is fixed) or $n_{e}^{2} T^{1 / 2} \propto(1+z)^{5 / 2}=2.8$ (if the comoving size of a cluster is fixed). Since the cluster size is not fixed either in comoving size or in physical size, the truth is probably interme-

TABLE 1B

Parameters of Schechter Fits for the X-Ray Cluster Luminosity FUNCTION IN THE 0.3-3.5 keV BAND

\begin{tabular}{|c|c|c|c|c|c|c|c|}
\hline $\begin{array}{c}z \\
(1)\end{array}$ & $\begin{array}{c}\alpha \\
(2)\end{array}$ & $\begin{array}{l}L_{x}^{*} \\
(3)\end{array}$ & $\begin{array}{l}n_{0} \\
(4)\end{array}$ & $\begin{array}{c}n\left(L>10^{43}\right) \\
(5)\end{array}$ & $\begin{array}{c}n\left(L>10^{44}\right) \\
(6)\end{array}$ & $\begin{array}{l}j_{\mathrm{cl}} \\
(7)\end{array}$ & $\begin{array}{l}j_{\text {gas }} \\
(8)\end{array}$ \\
\hline \multicolumn{8}{|c|}{ X-Ray Cluster Core Luminosity $\left(<0.5 h^{-1} \mathrm{Mpc}\right)$} \\
\hline $0 \ldots \ldots \ldots \ldots \ldots$ & 1.41 & 0.25 & 11.9 & 4.04 & $1.0 \times 10^{-2}$ & 0.045 & 0.16 \\
\hline $0.2 \ldots \ldots \ldots \ldots \ldots$ & 1.39 & 0.27 & 20.0 & 8.40 & $6.0 \times 10^{-2}$ & 0.079 & 0.24 \\
\hline $0.5 \ldots \ldots \ldots \ldots \ldots$ & 1.43 & 0.28 & 22.6 & 9.91 & $2.8 \times 10^{-2}$ & 0.099 & 0.30 \\
\hline $0.7 \ldots \ldots \ldots \ldots$ & 1.34 & 0.15 & 56.1 & 13.2 & $8.0 \times 10^{-2}$ & 0.11 & 0.33 \\
\hline $1 \ldots \ldots \ldots \ldots \ldots$ & 1.34 & 0.14 & 65.3 & 13.3 & $1.6 \times 10^{-2}$ & 0.12 & 0.34 \\
\hline $2 \ldots \ldots \ldots \ldots \ldots$ & 1.41 & 0.15 & 41.0 & 6.73 & $4.5 \times 10^{-2}$ & 0.093 & 0.25 \\
\hline & 1.31 & 0.089 & 78.3 & 2.53 & $2.2 \times 10^{-4}$ & 0.091 & 0.19 \\
\hline $5 \ldots \ldots \ldots \ldots \ldots \ldots \ldots$ & 1.65 & 0.14 & 2.92 & $3.0 \times 10^{-5}$ & $1.0 \times 10^{-7}$ & 0.010 & 0.019 \\
\hline \multicolumn{8}{|c|}{ X-Ray Cluster Total Luminosity $\left(<1 h^{-1} \mathrm{Mpc}\right)$} \\
\hline $0 \ldots \ldots \ldots \ldots \ldots$ & 1.36 & 0.64 & 13.2 & 11.4 & 0.40 & 0.12 & 0.16 \\
\hline $0.2 \ldots \ldots \ldots \ldots \ldots$ & 1.33 & 0.60 & 23.3 & 18.5 & 0.61 & 0.19 & 0.24 \\
\hline $0.5 \ldots \ldots \ldots \ldots \ldots$ & 1.36 & 0.53 & 28.8 & 21.1 & 0.51 & 0.21 & 0.30 \\
\hline $0.7 \ldots \ldots \ldots \ldots \ldots$ & 1.29 & 0.32 & 58.7 & 25.2 & 0.19 & 0.24 & 0.33 \\
\hline $1 \ldots \ldots \ldots \ldots \ldots$ & 1.29 & 0.30 & 66.8 & 26.8 & 0.17 & 0.26 & 0.34 \\
\hline $2 \ldots \ldots \ldots \ldots \ldots \ldots$ & 1.31 & 0.24 & 59.2 & 18.6 & 0.049 & 0.19 & 0.25 \\
\hline $3 \ldots \ldots \ldots \ldots \ldots$ & 1.33 & 0.20 & 53.1 & 13.3 & 0.015 & 0.14 & 0.19 \\
\hline $5 \ldots \ldots \ldots \ldots \ldots$ & 1.58 & 0.23 & 3.56 & 1.14 & $1.5 \times 10^{-3}$ & 0.017 & 0.019 \\
\hline
\end{tabular}


TABLE 1C

Parameters of Schechter Fits for the X-Ray Cluster Luminosity FUNCTION IN THE 0.5-4.5 keV BAND

\begin{tabular}{|c|c|c|c|c|c|c|c|}
\hline $\begin{array}{c}z \\
(1)\end{array}$ & $\begin{array}{c}\alpha \\
(2)\end{array}$ & $\begin{array}{l}L_{x}^{*} \\
(3)\end{array}$ & $\begin{array}{l}n_{0} \\
\text { (4) }\end{array}$ & $n\left(L>10^{43}\right)$ & $\begin{array}{c}n\left(L>10^{44}\right) \\
(6)\end{array}$ & $\begin{array}{l}j_{\mathrm{cl}} \\
(7)\end{array}$ & $\begin{array}{c}j_{\text {gas }} \\
(8)\end{array}$ \\
\hline \multicolumn{8}{|c|}{ X-Ray Cluster Core Luminosity $\left(<0.5 h^{-1} \mathrm{Mpc}\right)$} \\
\hline 0 . & 1.36 & 0.23 & 14.1 & 4.26 & $8.5 \times 10^{-3}$ & 0.046 & 0.18 \\
\hline 0.2 . & 1.34 & 0.27 & 21.0 & 8.61 & $7.9 \times 10^{-2}$ & 0.077 & 0.24 \\
\hline $0.5 \ldots \ldots \ldots \ldots$ & 1.37 & 0.26 & 25.4 & 8.94 & $2.9 \times 10^{-2}$ & 0.094 & 0.29 \\
\hline $0.7 \ldots \ldots \ldots \ldots$ & 1.31 & 0.16 & 52.8 & 12.0 & $9.2 \times 10^{-2}$ & 0.11 & 0.31 \\
\hline & 1.30 & 0.15 & 61.8 & 10.4 & $1.0 \times 10^{-2}$ & 0.12 & 0.31 \\
\hline$\ldots \ldots \ldots$ & 1.39 & 0.17 & 33.3 & 5.43 & $4.9 \times 10^{-2}$ & 0.083 & 0.20 \\
\hline $3 \ldots \ldots \ldots \ldots \ldots$ & 1.31 & 0.081 & 60.1 & 0.89 & $1.0 \times 10^{-5}$ & 0.064 & 0.13 \\
\hline $5 \ldots \ldots \ldots \ldots \ldots$ & 1.65 & 0.10 & 1.6 & 0.11 & $1.0 \times 10^{-7}$ & 0.004 & 0.010 \\
\hline \multicolumn{8}{|c|}{ X-Ray Cluster Total Luminosity $\left(<1 h^{-1} \mathrm{Mpc}\right)$} \\
\hline $0 \ldots$ & 1.34 & 0.68 & 12.7 & 11.3 & 0.45 & 0.12 & 0.18 \\
\hline $0.2 \ldots \ldots \ldots \ldots \ldots$ & 1.32 & 0.82 & 19.0 & 19.1 & 1.06 & 0.21 & 0.24 \\
\hline $0.5 \ldots \ldots \ldots \ldots$ & 1.32 & 0.54 & 29.2 & 21.0 & 0.57 & 0.21 & 0.29 \\
\hline $0.7 \ldots \ldots \ldots \ldots$ & 1.26 & 0.33 & 55.8 & 24.4 & 0.22 & 0.23 & 0.31 \\
\hline $1 \ldots \ldots \ldots \ldots \ldots$ & 1.26 & 0.31 & 61.7 & 25.3 & 0.18 & 0.24 & 0.31 \\
\hline $2 \ldots \ldots \ldots \ldots \ldots$ & 1.32 & 0.31 & 38.1 & 16.1 & 0.11 & 0.16 & 0.20 \\
\hline $3 \ldots \ldots \ldots \ldots \ldots$ & 1.32 & 0.22 & 37.4 & 10.6 & 0.019 & 0.11 & 0.13 \\
\hline $5 \ldots \ldots \ldots \ldots \ldots$ & 1.58 & 0.34 & 1.35 & 0.72 & $3.9 \times 10^{-3}$ & 0.0097 & 0.010 \\
\hline
\end{tabular}

diate between these two numbers. The average of these two numbers is about 6 , which is consistent with our preceding extrapolation. Alternatively phrased, a model with $\sigma_{8} \approx$ 0.6-0.7 and the same values of $\left(H_{0}, \Omega_{b}\right)$ would have produced approximately the correct X-ray luminosity function.

This result - that standard CDM, as normalized to $C O B E$ on large scales, has too much power on small scales by factors of $1.5-2.0$-is by now well known (cf. Davis et al. 1992). The results of this paper confirm the common knowledge using a new technique and a new observational measure. This conclusion would be greatly strengthened if we were to use the observed ratio of gas to total mass in the X-ray-emitting regions rather than relying, as we have, on light-element nucleosynthesis for the ratio $\rho_{b} / \rho_{\text {tot }}$. Following White (1991), we would have (for $h=0.5$ ) taken $\rho_{\text {gas }} / \rho_{\text {tot }}=0.13$ rather than the value we derived, 0.04 . Using the larger, observed value, our luminosities would have been larger by a factor of about 10.

The value of $\alpha$ found in our work for the Schechter parameter of approximately $1.4-1.5$ is slightly smaller than the bestfit observational values $1.9-2.0$ quoted by Henry (1992). This difference, as can be noted visually in our Figure $4 b$, is probably not significant. The values of $\alpha$ quoted in Table 1 are primarily determined by lower luminosities than those used in the observational fits. In overlapping luminosity ranges the computed and observed values of $\alpha$ are in adequate agreement.

TABLE 1D

PARAMETERS OF SCHECHTER FItS FOR THE X-RAY Cluster LuminOSITY FUNCTION IN THE 2-10 keV BAND

\begin{tabular}{|c|c|c|c|c|c|c|c|}
\hline $\begin{array}{c}z \\
(1)\end{array}$ & $\begin{array}{c}\alpha \\
(2)\end{array}$ & $\begin{array}{l}L_{x}^{*} \\
(3)\end{array}$ & $\begin{array}{l}n_{0} \\
(4)\end{array}$ & $\begin{array}{c}n\left(L>10^{43}\right) \\
(5)\end{array}$ & $\begin{array}{c}n\left(L>10^{44}\right) \\
(6)\end{array}$ & $\begin{array}{l}j_{\mathrm{cl}} \\
(7)\end{array}$ & $\begin{array}{c}j_{\text {gas }} \\
(8)\end{array}$ \\
\hline \multicolumn{8}{|c|}{ X-Ray Cluster Core Luminosity $\left(<0.5 h^{-1} \mathrm{Mpc}\right)$} \\
\hline $0 \ldots \ldots \ldots \ldots \ldots$ & 1.33 & 0.87 & 6.26 & 6.65 & 0.39 & 0.073 & 0.18 \\
\hline $0.2 \ldots \ldots \ldots \ldots \ldots$ & 1.27 & 0.35 & 15.4 & 12.0 & 0.50 & 0.068 & 0.21 \\
\hline $0.5 \ldots \ldots \ldots \ldots$ & 1.28 & 0.30 & 18.8 & 13.5 & 0.56 & 0.071 & 0.29 \\
\hline $0.7 \ldots \ldots \ldots \ldots$ & 1.29 & 0.29 & 21.3 & 16.6 & 0.35 & 0.079 & 0.21 \\
\hline $1 \ldots \ldots \ldots \ldots \ldots$ & 1.28 & 0.24 & 25.9 & 17.6 & 0.32 & 0.079 & 0.18 \\
\hline $2 \ldots \ldots \ldots \ldots \ldots$ & 1.46 & 0.70 & 3.69 & 7.31 & 0.057 & 0.042 & 0.080 \\
\hline $3 \ldots \ldots \ldots \ldots \ldots$ & 1.30 & 0.05 & 20.0 & 2.43 & 0.020 & 0.013 & 0.026 \\
\hline $5 \ldots \ldots \ldots \ldots \ldots$ & 1.47 & 0.018 & 0.51 & $1.8 \times 10^{-3}$ & $1.0 \times 10^{-7}$ & $1.5 \times 10^{-4}$ & $7.6 \times 10^{-4}$ \\
\hline \multicolumn{8}{|c|}{ X-Ray Cluster Total Luminosity $\left(<1 h^{-1} \mathrm{Mpc}\right)$} \\
\hline $0 \ldots \ldots \ldots \ldots \ldots$ & 1.28 & 2.84 & 6.45 & 13.2 & 2.43 & 0.23 & 0.18 \\
\hline $0.2 \ldots \ldots \ldots \ldots \ldots$ & 1.23 & 0.72 & 17.0 & 14.1 & 0.75 & 0.15 & 0.21 \\
\hline $0.5 \ldots \ldots \ldots \ldots \ldots$ & 1.25 & 0.64 & 19.0 & 14.7 & 0.62 & 0.15 & 0.29 \\
\hline $0.7 \ldots \ldots \ldots \ldots \ldots$ & 1.28 & 0.80 & 16.5 & 15.6 & 0.89 & 0.17 & 0.21 \\
\hline $1 \ldots \ldots \ldots \ldots \ldots$ & 1.26 & 0.53 & 24.2 & 16.3 & 0.47 & 0.16 & 0.18 \\
\hline $2 \ldots \ldots \ldots \ldots \ldots$ & 1.34 & 0.38 & 11.0 & 5.78 & 0.067 & 0.057 & 0.080 \\
\hline $3 \ldots \ldots \ldots \ldots \ldots$ & 1.26 & 0.10 & 16.0 & 1.37 & $1.6 \times 10^{-5}$ & 0.020 & 0.026 \\
\hline $5 \ldots \ldots \ldots \ldots \ldots$ & 1.27 & 0.020 & 1.80 & $5.6 \times 10^{-4}$ & $1.9 \times 10^{-22}$ & $4.5 \times 10^{-4}$ & $7.6 \times 10^{-4}$ \\
\hline
\end{tabular}


The number density of cluster peaks at intermediate redshift and the typical luminosity is (for small redshift) relatively constant, so there is a peak emissivity at approximately $z=0.8$ for the several $\mathrm{keV}$ bands. Thus, crudely speaking, in this model one expects "positive" evolution until nearly $z=1$, and negative evolution thereafter. But the situation is more complex.

Let us first look at columns (8) and (9) of Table 1A, which show the evolution of the total bremsstrahlung emissivity per unit comoving volume and the integrated cluster emissivity. The tabulated evolution for these two measures is roughly parallel, indicating that the brightest clusters emit a roughly constant fraction of the total bremsstrahlung radiation. The peak, seen at redshift $z=0.5-1.0$, is at first surprising. The Press-Schechter treatment indicates that several effects are at work. The fraction of mass in deep potential wells can only increase with time, and once gas is virialized, its density (metric, not comoving) does not change, but merging of clusters will reduce the typical density without affecting the temperature.

We believe that the peak seen in emission at moderate redshift is real. Clusters form as a result of a hydrodynamic shock, go through a phase of maximum density, and then reexpand somewhat to reach an equilibrium system with somewhat lower density. The approximate Press-Schechter formalism, which does not allow for this overshooting, does not include this effect. In addition, the peak kinetic energy density occurs, for the CDM power spectrum, for wavelengths of order $30 h^{-1}$ $\mathrm{Mpc}$, which became nonlinear at $z \sim 0.5$ with our chosen normalization. In short, it is not quantitatively accurate to treat the clusters as derived from a power-law form of $\boldsymbol{P}_{\boldsymbol{k}}$ and as in exact virial equilibrium. Both ram-pressure confinement and subsequent expansion are also important phenomena, as well as the merging phenomena treated by the Press-Schechter formalism.

The self-similar evolution adopted in the semianalytic treatment of Kaiser (1986; see also Blanchard et al. 1992) predicts an emissivity scaling as $(1+z)^{5 / 2}$ for moderate redshifts. This is a far stronger evolution than shown in Table 1A (bottom panel), columns (8) and (9). These show a positive evolution to $z=1$ by a factor of about 1.6 , whereas the Kaiser prediction is a factor of 5.7. Thus, it seems that detailed numerical simulations, such as those in the present work, will be required to permit meaningful comparisons between the observed and theoretically predicted evolution of the cluster X-ray luminosity function. A conclusive test of the argument presented here could be made if we were to repeat the simulation with an assumed power-law form for $\boldsymbol{P}_{k}$. Then self-similar results should be obtained. This test is in progress.

While the integrated X-ray emissivity evolves fairly slowly over the period surveyed in Table $1 \mathrm{~A}$, both $L_{*}$ and $n_{0}$ evolve more rapidly and in opposite directions, with more and more lower and lower luminosity clusters at higher redshifts. To highlight the negative evolution of the bright end of the luminosity function, we computed columns (5) and (6) of Tables 1B-1D (cols. [6] and [7] of Table 1A), the comoving density of clusters having luminosity greater than $10^{43}$ and $10^{44}$ ergs s $^{-1}$. For reasons stated earlier (based on our limited box size), we use the Schechter fit rather than direct counts to compute these columns. Comparing columns (4) and (6) of Tables 1B-1D (cols. [5] and [7] of Table 1A), we see that, although the total number density $n_{0}$ of clusters increases with redshift (until $z=3$ ), the number density of high-luminosity clusters decreases after $z=0.5$. This is presumably one of the effects leading to the observational appearance of "negative evolution" (i.e., fewer bright clusters at higher redshifts). Statistical fluctuations in our results are still quite significant owing to the limited box size.

Redshift effects strongly exaggerate this tendency to observe negative evolution, since higher redshift clusters tend to have lower temperatures (cf. col. [4] of Table 1A, and Figs. 5 and 12) and both effects can shift the observed $(z=0)$ spectrum out of the high-energy $\mathrm{X}$-ray bands. Note that the negative evolution in the density of clusters with $L>10^{44} \mathrm{ergs} \mathrm{s}^{-1}$ is steeper and steeper going from Table 1A to Table 1D, as one looks at higher energy bands.

The emission-weighted temperature, $T_{x}$, of each cluster is calculated, and the distributions are shown in Figure 5. The arrow in each panel indicates the luminosity-weighted average at the given epoch. We see that at all epochs the coolest clusters dominate the statistics (the turnover at low $T_{x}$ is presumably caused by our definition of minimum cell luminosity to constitute an X-ray-bright cell), but the mean is determined by the high-mass, high-luminosity, high-temperature end of the distribution. The increase of the mean with increasing time is for the usual reason, $T_{x} \propto(H \lambda)^{2}$, with the wavelength of nonlinear waves increasing with time faster than $H$ (Hubble constant) decreases. The mean temperatures, indicated by arrows, are included in column (3) of Table 1A. Looking ahead to Figure 7 (top panels), we see the strong correlation found between $T_{x}$ and total luminosity (clusters are shown at $z=0$ ).

Now let us turn again to the total cluster luminosity (versus core luminosity) as shown in the lower panels of the tables and in panels $b$ of Figures 1-6 and the bottom panels of Figure 7, the quantity normally measured by satellite observations. The decline in numbers of bright clusters with redshift is steeper for the total than for the cores, because the high-redshift clusters are physically smaller.

We can also roughly estimate the effective radii of the clusters by assuming that the emission has a profile

$$
j=\frac{j_{0}}{\left[1+\left(r / r_{x}\right)^{2}\right]^{2}}
$$

and determining, from the ratio of the luminosity of the central cell to the total cluster luminosity, the value of $r_{x}$ which would produce this ratio. We show in Figure 6 the radii determined in this fashion. The peaks seen in the panels of Figure 6 are, of course, artificially induced by our nominal resolution limit of $0.31 h^{-1} \mathrm{Mpc}$, but the distribution to larger radii should be reasonably accurate. Arrows indicate the luminosity-weighted average values. Since brighter clusters tend to be resolved, these numbers should be reliable. We see a weak trend of increasing size with increasing time, which is in the theoretically anticipated direction. Longer wavelengths become nonlinear later, producing larger clusters, and smaller clusters merge to produce larger clusters with increasing time.

Now in Figures $7 a$ and $7 b$ we show the scatter plots of $\left(T_{x}, r_{x}\right)$ versus $L_{\text {tot }}$. We see that there is a clear correlation between $L$ and $T_{x}$. The best straight-line fit shown as a dashed line corresponds to slopes of 0.39 and 0.37 , respectively. The solid line indicates the observation (Henry 1992). But we do not see any correlation between $L$ and $r_{x}$. These two correlations imply (since $L_{x} \propto T_{x}^{1 / 2} r_{x}^{3} \rho^{2}$ ) that $\rho$ is an increasing function of $J_{v}\left(\rho \sim T_{x}\right)$. This is expected, since higher density peaks contain more energy and therefore produce stronger shocks. The predicted temperatures are higher than those 

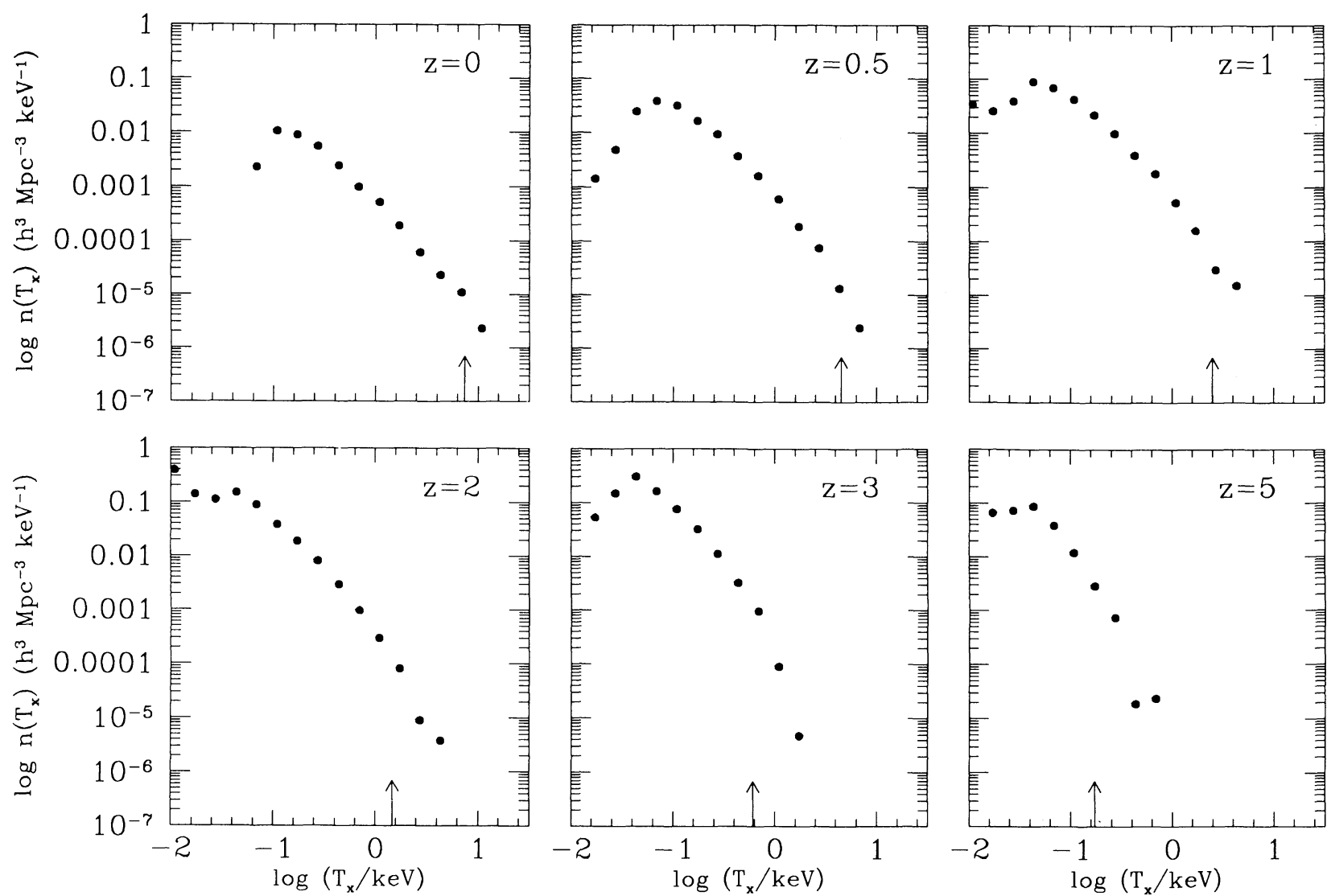

Fig. $5 a$
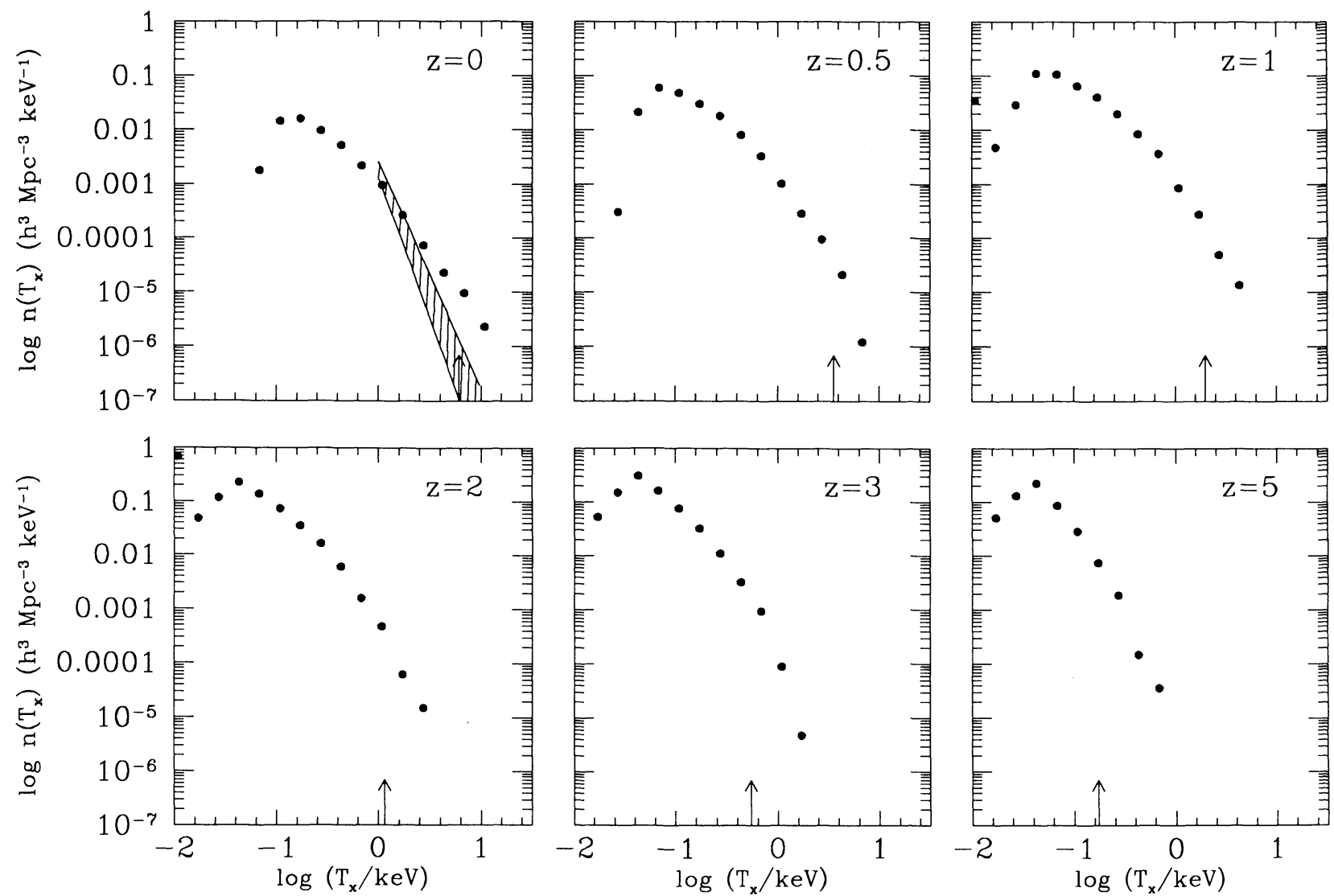

FIG. $5 b$

Fig. 5. - X-ray cluster temperature ( $T_{x}$, emission-weighted temperature) distribution function at six different redshifts, $z=(0,0.5,1,2,3,5)$. Arrows indicate the luminosity-weighted average temperature $\bar{T}_{x}$ at each epoch. As before, core and total cluster properties are shown in $(a)$ and $(b)$, respectively. In the first $(z=0)$ panel in $(b)$ the hatched area is the observed temperature function from Henry \& Arnaud (1991) $\left[\left(1.8_{-0.5}^{+0.8} \times 10^{-3} h^{3} \mathrm{Mpc}^{-3} \mathrm{keV}^{-1}\right)(k T)^{-4.7 \pm 0.5}\right]$. The artificial turnover at low $T_{x}$ does not affect our luminosity-weighted results. 

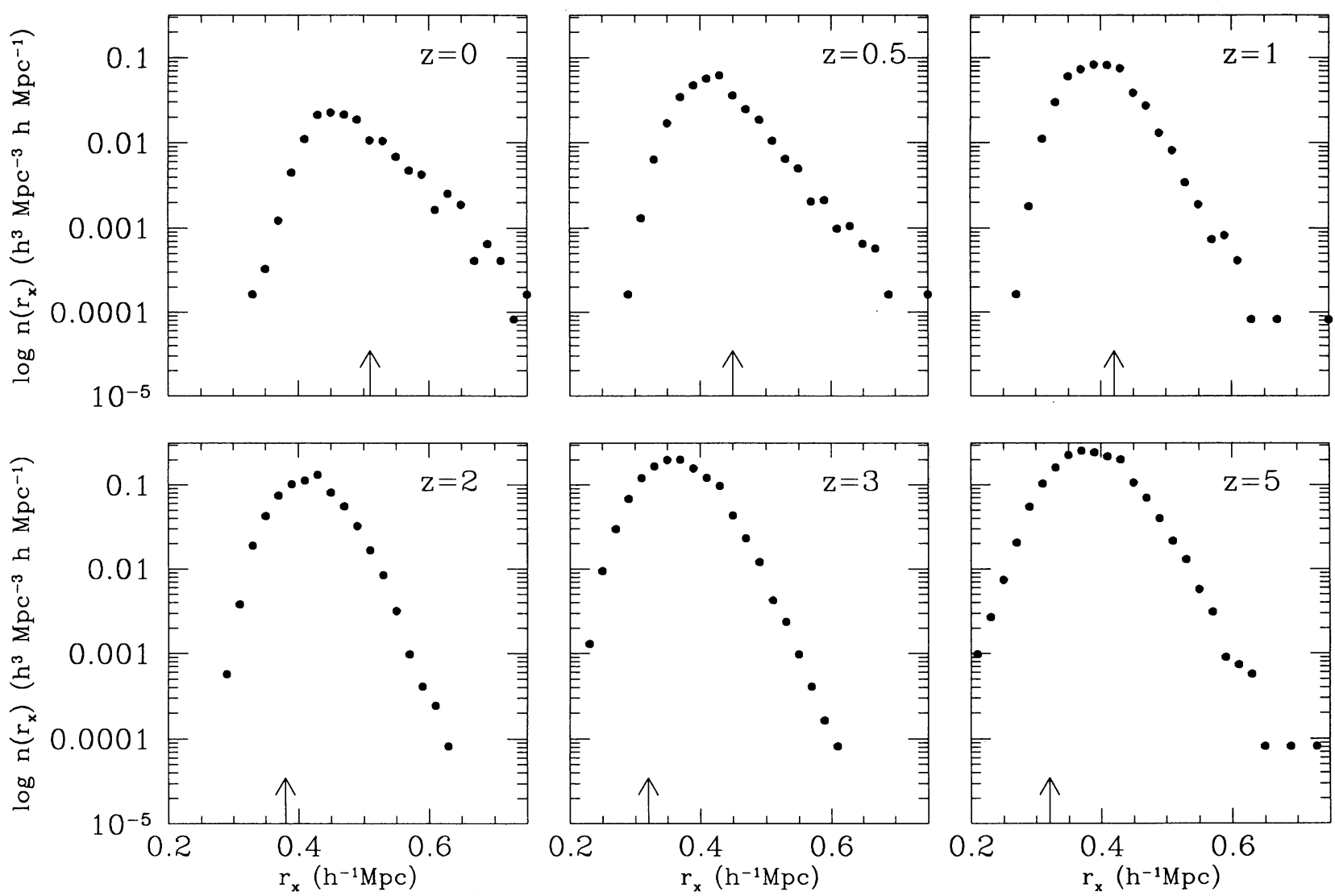

Fig. $6 a$
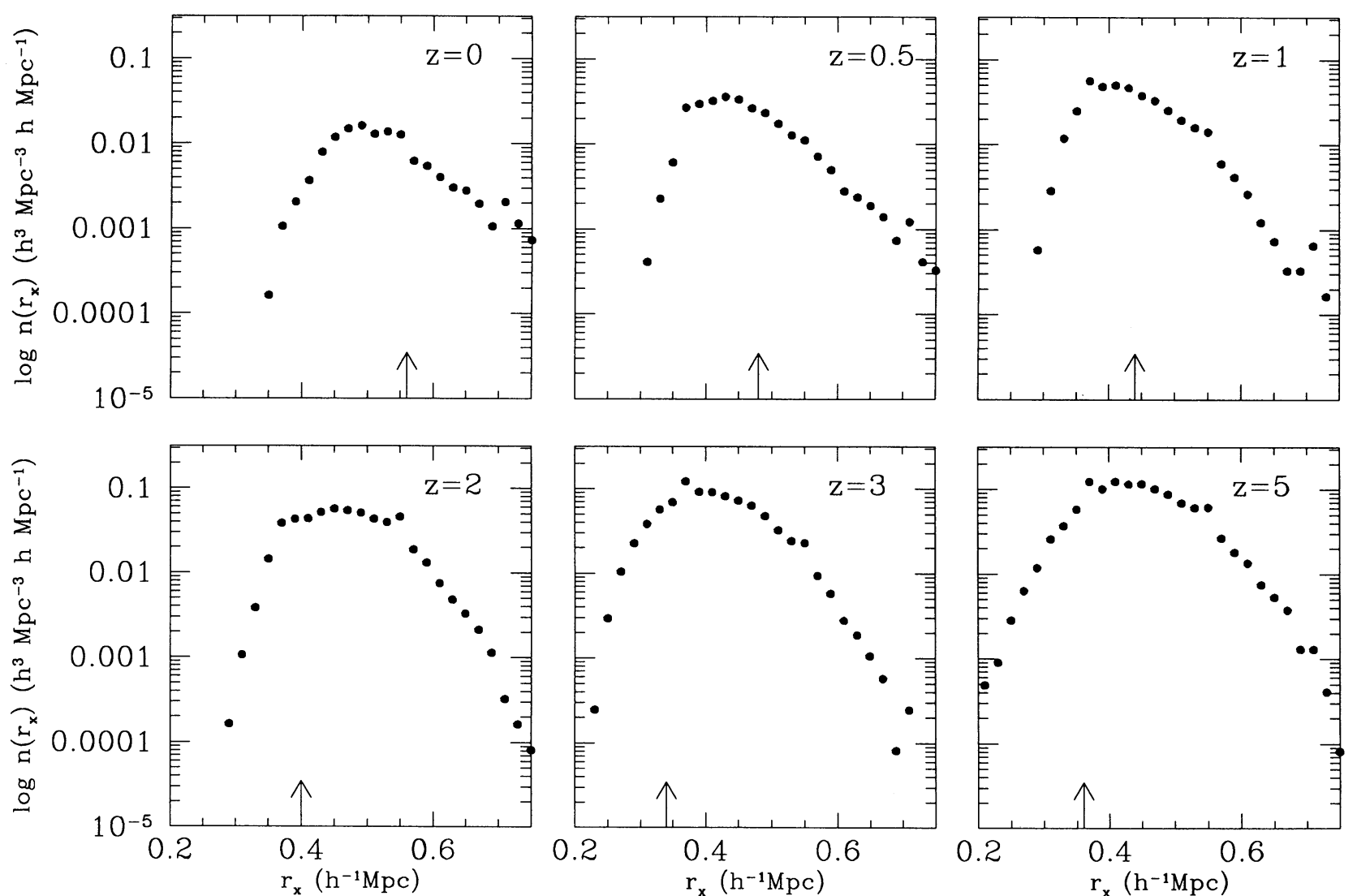

Fig. $6 b$

FiG. 6.-X-ray cluster effective radius $\left(r_{x}\right)$ distribution (cf. eq. [4]). Arrows indicate the luminosity-weighted average values at each epoch. As before, $(a)$ and $(b)$ show core and total cluster properties, respectively. The artificial turnover at low $r_{x}$ indicates resolution problem with our numerical methods and probably does affect our luminosity-weighted results. 


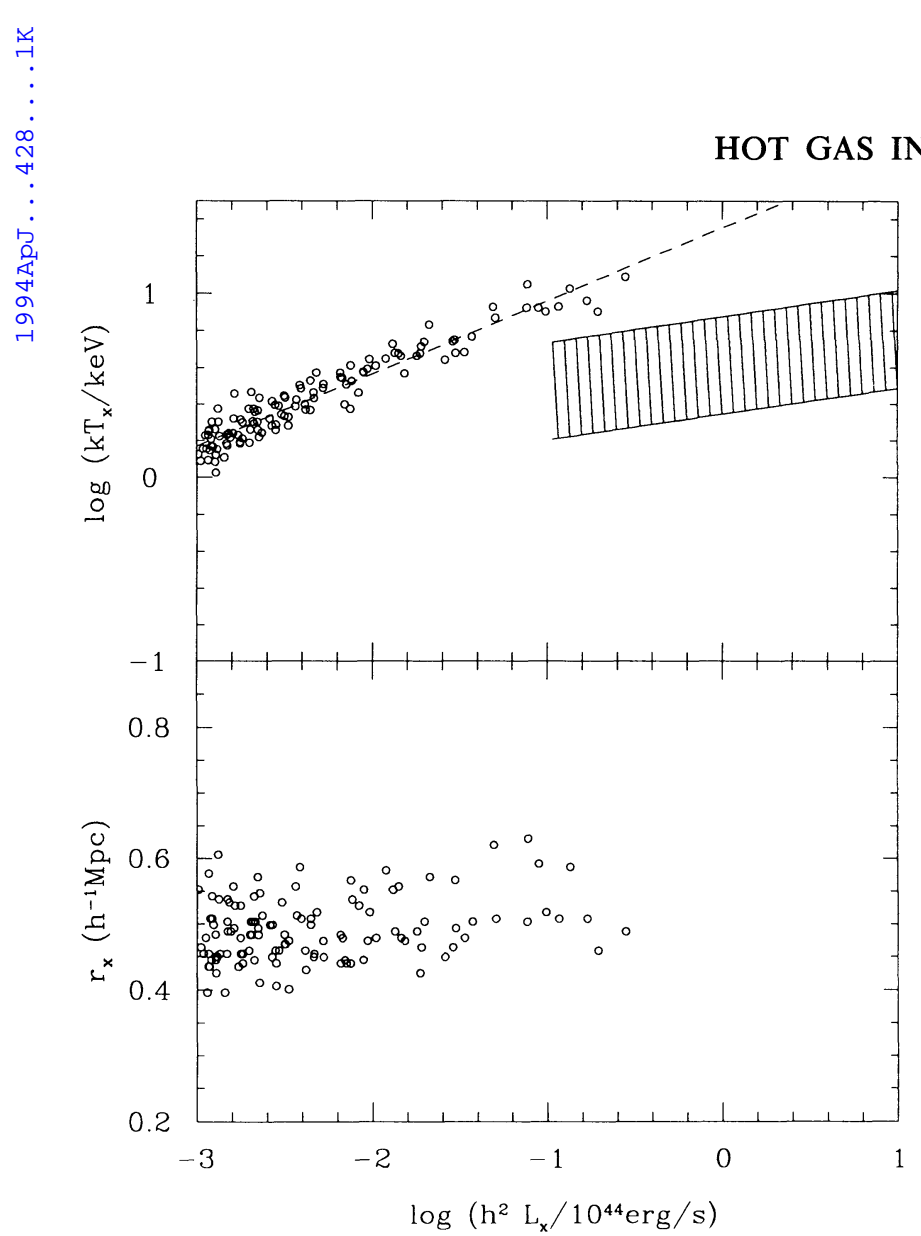

FIG. $7 a$

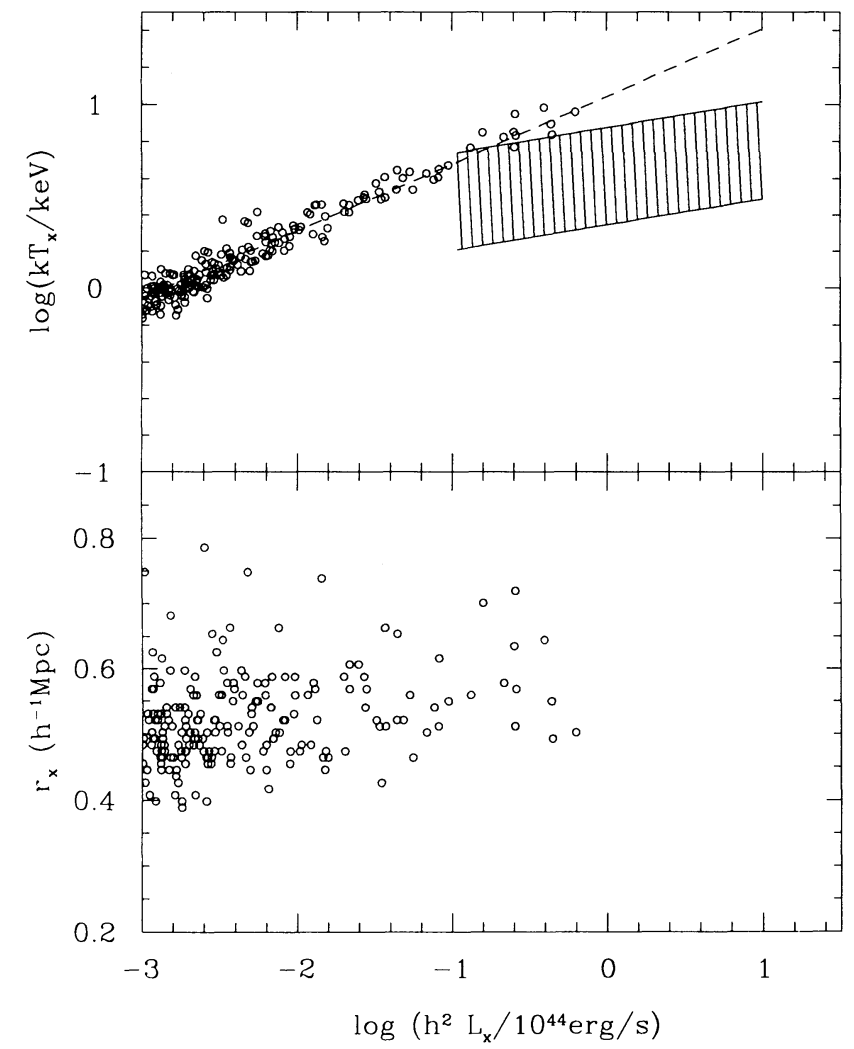

FIG. $7 b$

FIG. 7.-Top panels: Scatter plots of $T_{x}$ vs. $L_{\text {tot }}$ at $z=0$. Bottom panels: Scatter plots of $r_{x}$ vs. $L_{\text {tot }}$ at $z=0$; no trend is seen. The dashed lines in the upper panels

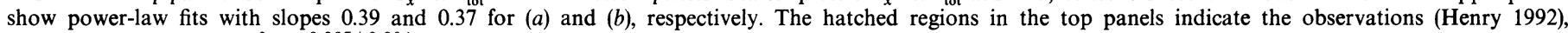

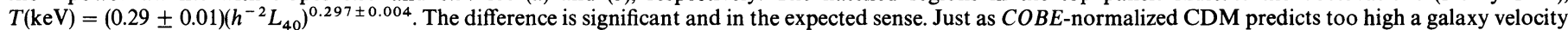
dispersion, so it predicts too-high cluster X-ray temperatures.

observed, and the difference is probably significant and in the expected sense. Just as $C O B E$-normalized CDM predicts too high a galaxy velocity dispersion, so it predicts too-high cluster $\mathrm{X}$-ray temperatures.

Finally, we address temperature variations within clusters. Given our limited resolution, there is little that can be accurately determined from our simulations, but we are able to compare the central cell (volume $=3.1 \times 10^{-2} h^{-3} \mathrm{Mpc}^{3}$ ) with the surrounding cells $\left(3^{3}-1^{3} ;\right.$ volume $\left.=5.9 \times 10^{-1} h^{-3} \mathrm{Mpc}^{3}\right)$ and the cells surrounding these cells $\left(5^{3}-3^{3}\right.$; volume $=3.0 h^{-3}$ $\left.\mathrm{Mpc}^{3}\right)$. We define the ratio of the inner cell to the next shell as $T_{c} / T_{\text {shell }}$ and show the scatter diagram in Figure 8 . No trend is seen with luminosity, and the median value, indicated by the solid line, is $1.3-1.4$. The cluster gas deviates significantly from isothermality with a $30 \%$ temperature decline typically found by a radius of $0.4-0.5 h^{-1} \mathrm{Mpc}$. In Figure 9 we compare the temperatures found in the three regions noted above and normalized to the temperature in the central cell. The large dispersion is indicated by the error bars.

In the development of a one-dimensional Zel'dovich pancake (Shapiro \& Struck-Marcell 1985; Ryu et al. 1993) there is a temperature minimum at the center of a pancake, and we might expect, on theoretical grounds, that a similar effect should occur in real three-dimensional clusters. (The reason is that the gas, which is shocked first, is put on a low adiabat and should maintain a lower temperature and higher density than surrounding gaseous elements with which it is in pressure equilibrium.) In the paper by Bryan et al. (1994a) we can see pic- torial evidence for such shallow minima. The shallow temperature gradient of the opposite sign seen in Figures 8 and 9 is, we believe, not inconsistent with these theoretical and pictorial expectations, due to the crude averaging used in producing the above figures.

Let us turn away from the consideration of specifically identified clusters to ask a more general question concerning the

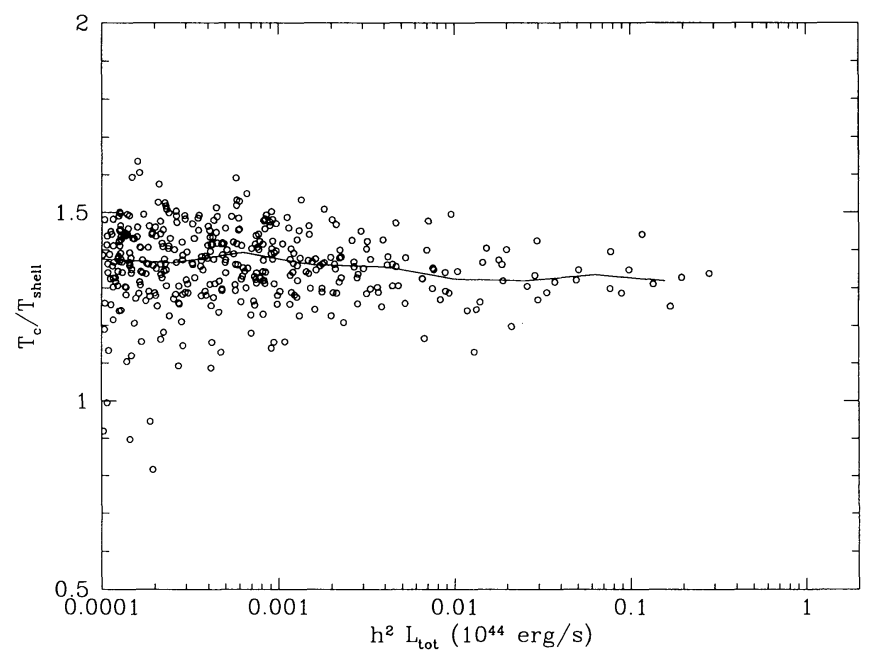

FIG. 8.-Ratio of the central cell temperature to the temperature of its surrounding shell ( $\sim 1$ cell thick) as a function of $L_{\mathrm{tot}} ;$ no trend is seen. 


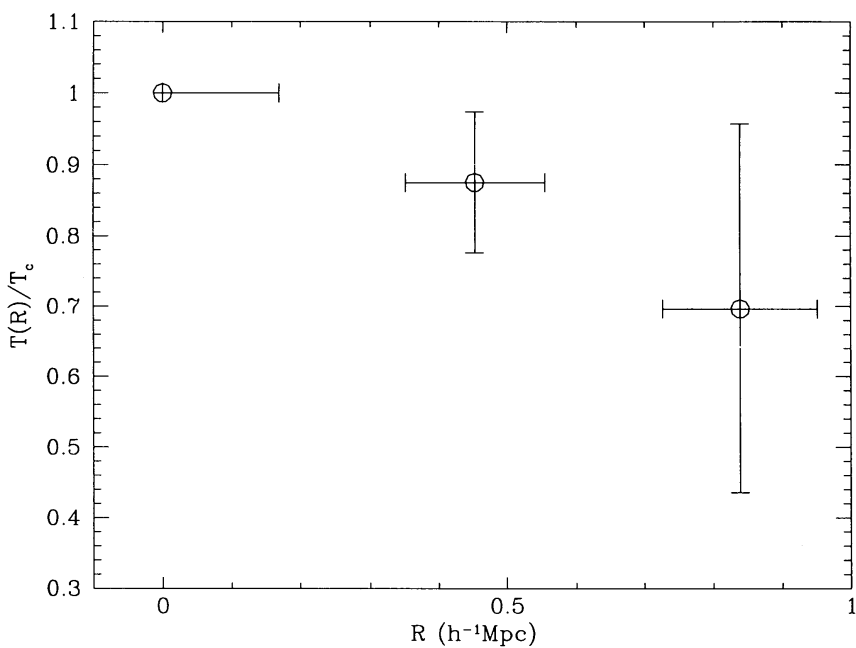

Fig. 9.-Comparison of the temperatures found in the three regions (central cell, the shell surrounding the central cell, and the next outer shell) and normalized to the temperature in the central cell. A moderate decline of temperature with radius is found, but there is considerable variance in the relationship.

hot gas. Is it fairly represented in dense regions, or is it "biased"-overrepresented-or antibiased? This is a question with great cosmological significance. If we know the ratio of gas (plus galaxies) to total matter in the clusters by direct observations, and we know, from light-element nucleosynthesis, the global baryon density, then we can divide the second number by the first to obtain the global matter density and compare with the cherished critical density. This line of argument has been carefully examined recently by White (1991) and also reanalyzed by Babul \& Katz (1993).

The argument depends on knowing whether or not $\rho_{b} / \rho_{\text {tot }}$ varies significantly from place to place and, in particular, whether this quantity will be found near its average value in the high-density regions where it can most easily be measured.

Our possibly counterintuitive results are shown in Figure 10, where we plot the ratio $\left(\rho_{\mathrm{IGM}} / \rho_{\text {tot }}\right)$ versus $\rho_{\text {tot }} /\left\langle\rho_{\text {tot }}\right\rangle$ smoothed

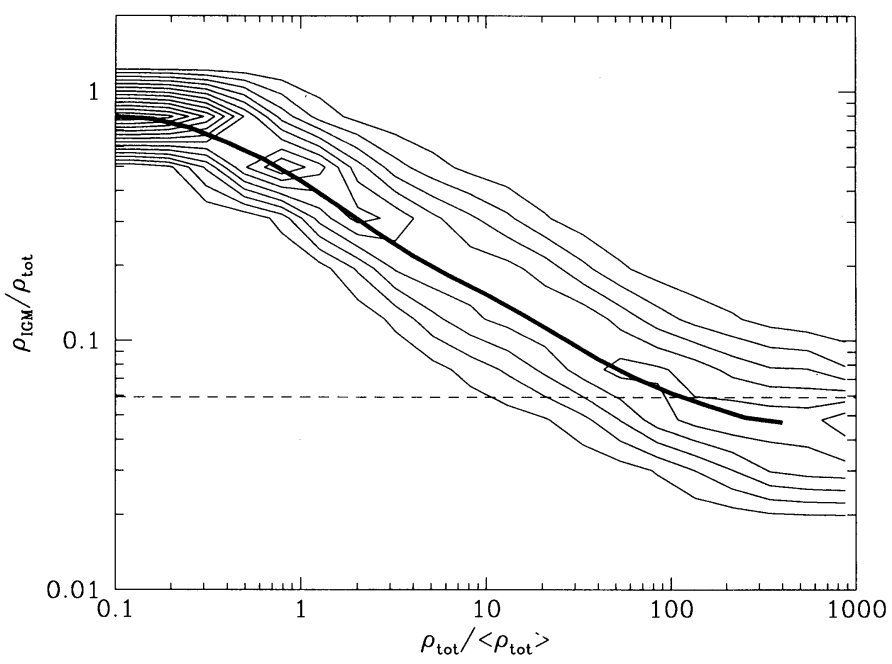

FIG. 10.-Ratio $\rho_{\text {IGM }} / \rho_{\text {tot }}$ as a function of $\rho_{\text {tot }} /\left\langle\rho_{\text {tot }}\right\rangle$. Results are smoothed by a Gaussian window of radius $1 h^{-1} \mathrm{Mpc}$. The global mean value of $\rho_{\mathrm{IGM}} / \rho_{\text {tot }}$ is shown by the dashed line. Note that in the highest density regions the gas is underrepresented, "antibiased," by a factor of about 1.7, whereas the voids are correspondingly gas-rich. by a Gaussian of radius $1 h^{-1} \mathrm{Mpc}$. At any value for the total density there is a wide range of possible values of $\rho_{\mathrm{IGM}}$, but the high-density regions actually have a lower than average ratio of baryons to total mass, whereas the void regions are gas-rich. In fact, in the regions (not shown) which have $\left(\rho_{\text {tot }} /\left\langle\rho_{\text {tot }}\right\rangle\right)<0.1$ (more than $90 \%$ underdense), which fill more than half of the box volume (filling fraction $51 \%$ ), the gas is relatively overdense by approximately a factor of 5 and constitutes $31 \%$ of the total mass as compared with the global average of $6 \%$. The cause of this segregation is primarily due to gas pressure, but is in part due to statistical effects: in low-density regions there will by chance be regions containing no dark matter particles. The trend shown, however, is real. After a collapse toward a shock/caustic the difference between the high-pressure inner region and the low-pressure, low-density regions outside the caustic causes the gas to expand outward at the speed of sound in an effort to achieve pressure equilibrium (recalling also that the universe as a whole is expanding). This effect, seen earlier in the one-dimensional calculations of Shapiro \& Struck-Marcell (1985), is also a partial cause of the peak in the cluster emissivity at $z \approx 1$. They reach maximum compression at that time and expand subsequently with consequent lowering of X-ray luminosity.

Thus, we believe that the phenomenon discovered in this high-resolution CDM simulation will be found to be generic. The X-ray clusters have a lower than average ratio of baryons to total mass. Evrard (1990) and all our previous work (with other codes and other scenarios) have shown the same result. If input from supernovae in cluster galaxies is important (cf. Yahil \& Ostriker 1973), as may be implied by the high metal abundance, then this statement is strengthened. The additional energy input from supernova winds can only contribute further to driving hot gas out of the clusters.

Even without this effect, we see that in the high-density clusters, where $\rho_{\text {tot }} /\left\langle\rho_{\text {tot }}\right\rangle$ approaches $10^{3}$, the gas is underrepresented by a factor of 1.7. Thus, White's (1991) conclusion that $\Omega$ is significantly less than unity is strengthened, and the estimated value of $\Omega$ is further reduced by this factor.

In our discussion of the bias or antibias of the gas, so far we have weighted all volumes equally. It would be more appropriate to look specifically at those regions within $h r<1 \mathrm{Mpc}$ at the centers of our X-ray clusters. Figure 11 shows just that. The histogram under the thin solid line shows the distribution of $\rho_{\text {gas }} / \rho_{\text {tot }}$ in our tabulated clusters with the median value of this quantity, 0.040 , indicated by the vertical thin solid line. But, since the observed sample is (approximately) picked on a luminosity-weighted basis, we constructed the histogram of $\rho_{\text {gas }} / \rho_{\text {tot }}$ using that weighting, and display it as the dotted region with median indicated by the dotted vertical thin line. The global (assumed) average for $\Omega_{b} / \Omega_{\text {tot }}$ is shown as the heavy vertical line. We see an antibias of 0.67 from the ratio of median to global value, which is reduced to 0.75 if we luminosity-weight the sample. Thus the effect mentioned, that clusters have a lower than average ratio of gas to total matter, withstands this more careful scrutiny. The heavy solid histogram indicating the observational situation is adapted from Jones \& Forman (1992). We see that the observed ratio is higher than the computed ratio by a factor of $2-3$.

\section{EVOLUTION OF THE X-RAY CLUSTERS: AN OBSERVATIONAL TEST FOR THE CDM SCENARIO?}

In Figures 1-4 we showed the evolution of the cluster luminosity function expected in the CDM scenario. In the easily 


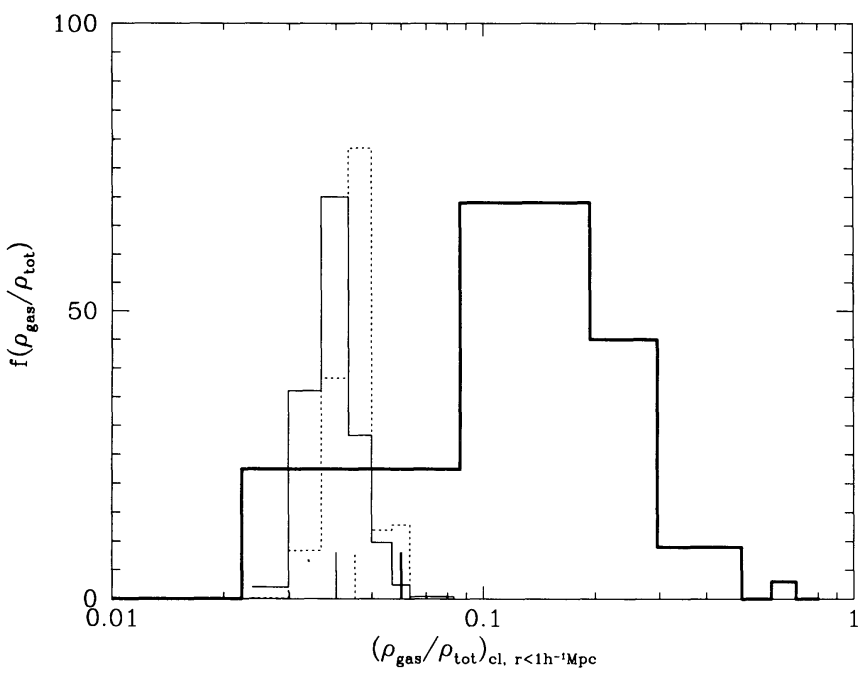

FIG. 11.-Thin solid line shows the distribution of $\rho_{\text {gas }} / \rho_{\text {tot }}$ in our tabulated clusters, with the median value of this quantity, 0.040 , indicated by the short vertical thin solid line. But, since the observed sample is (approximately) picked on a luminosity-weighted basis, we constructed the histogram of $\rho_{\text {gas }} / \rho_{\text {tot }}$ using that weighting and display it as the dotted region with median indicated by the short vertical thin dotted line. The global (assumed) average for $\Omega_{b} / \Omega_{\text {tot }}$ is shown as the short vertical heavy line. We see an antibias of 0.67 from the ratio of median to global value, which is reduced to 0.75 if we luminosity-weight the sample. The heavy solid histogram is the observation adapted from Jones \& Forman (1992); we see that the observed ratio is higher than that computed by a factor of $2-3$.

observed range of parameters $\left(0 \leq z \leq 1,10^{40}\right.$ ergs $\mathrm{s}^{-1} \leq$ $L_{x} \leq 10^{44}$ ergs s $^{-1}$ ), little evolution is seen in any of the computed bands aside from a decline in the number of brightest sources (somewhat uncertain due to our limited box size) and a modest increase (by about a factor of 2) in the luminosity function for fainter objects. The likely explanation for this has been mentioned; it is largely due to coincidental balancing of two effects: new breaking waves increase the luminosity density, but mergers decrease it. It just happens that for this spectrum at this epoch the net rate of change is small. In other scenarios we expect the results to be different, but only detailed calculations can prove this. As noted previously, our numerical

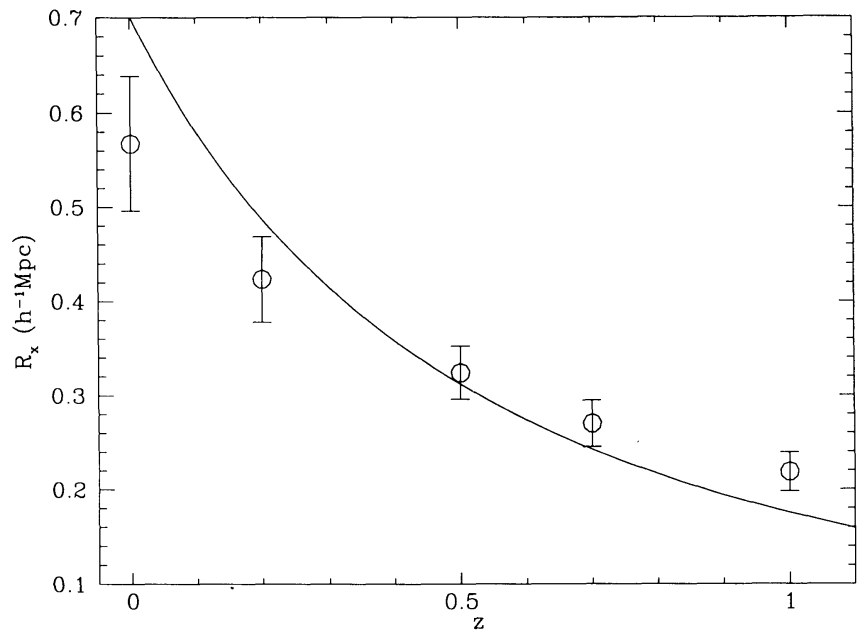

FIG. 12.-Average cluster core radii in physical units as a function of redshift for clusters with luminosity in the $0.5-4.5 \mathrm{keV}$ band greater than $10^{43} \mathrm{ergs}$ $\mathrm{s}^{-1}$. The best-fit evolution of the form $R_{x} \propto(1+z)^{-2}$ is shown as a solid curve.

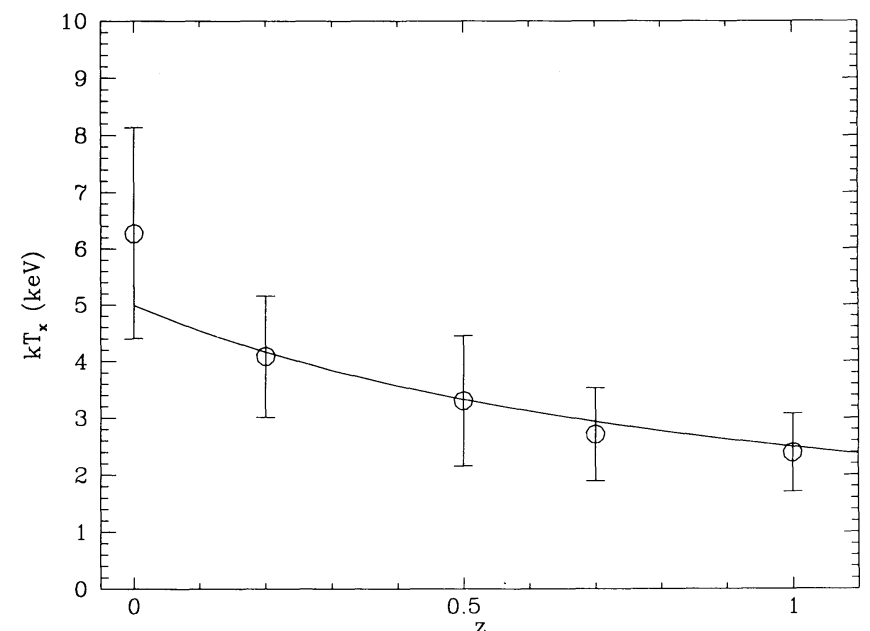

Fig. 13.-Average cluster temperature as a function of redshift for clusters with luminosity in the $0.5-4.5 \mathrm{keV}$ band greater than $10^{43} \mathrm{ergs} \mathrm{s}^{-1}$. The best-fit evolution of the form $T_{x} \propto(1+z)^{-1}$ is shown as a solid curve.

results contrast sharply with the semianalytic, approximate computations of Kaiser (1986) and Blanchard et al. (1992), who were required to make many simplifying assumptions, particularly concerning the cluster core radii. Kaiser (1986), assuming self-similar cluster evolution, predicted that the luminosity function would scale as $(1+z)^{5 / 2}$, in contrast to the minimal evolution displayed in our Figures 1-4.

However, Figures 5 and 6 did show substantial rates of change in other quantities, the temperatures and cluster radii, which are in better accord with prior expectations. These important trends are summarized in Figures 11 and 12, where

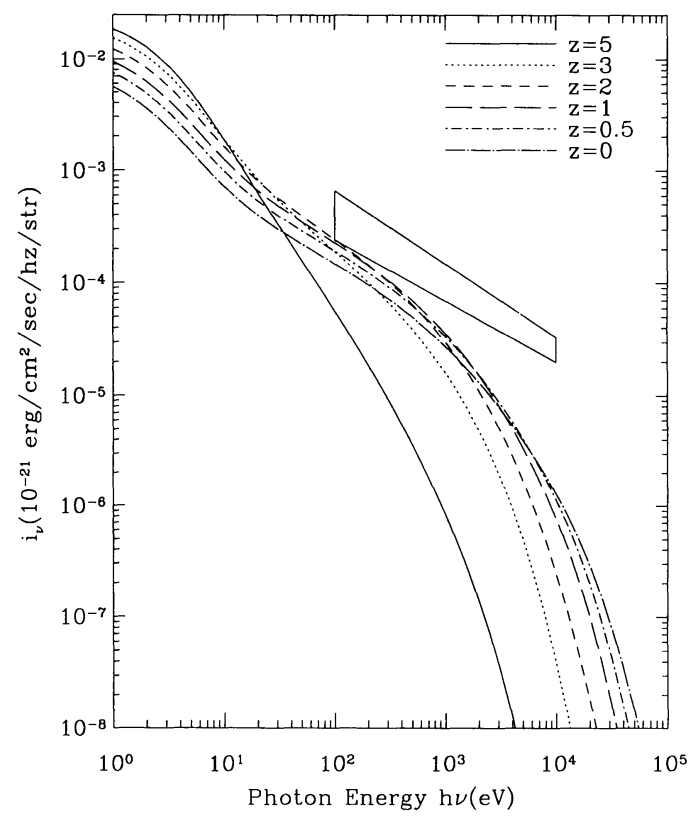

FIG. 14.-Mean radiation at six epochs, $z=5,3,2,1,0.5,0$. The box in the middle shows the observational data by Wu et al. (1991). Recall that no allowance is made in our calculation for line emission or absorption, with both important at energies less than $1 \mathrm{keV}$. This effect, numerical effects, and our too-low assumption of $\Omega_{b} / \Omega_{\text {tot }}$ (cf. Fig. 11) lead to a very significant underestimate of $i_{v}$, which is already in excess of what is permitted after removal of sources. 
we see a factor of 2-3 decline in both these quantities by redshift 1 and a 30\% decline in the (luminosity-weighted) mean temperature even in the small redshift range $z=0-0.2$. An analogous velocity dispersion decline was found by Frenk et al. (1990) in a dark-matter-only simulation which would correspond to a decline in $T_{x}$ with increasing redshift. We show as dashed lines in Figures 12 and 13 the best-fit curves analytically predicted by Kaiser: $R_{x} \propto(1+z)^{-2}$ and $T_{x} \propto(1+z)^{-1}$. Examination of these figures indicates that the radius changes less rapidly and the density more rapidly than theoretically expected. The trend of temperature with redshift should be detectable even with a relatively "soft" X-ray instrument such as $R O S A T$. It provides a test of $\Omega=1$ models, since in open, $\Omega<1$ models the structure will tend to freeze out at early epochs $(z \sim 1 / \Omega)$, with cluster temperatures not changing substantially after that time.

Figures 14 and 15 show the evolution in the background radiation field in two additional ways. The first shows what a comoving observer would have measured at various redshifts. Note the too-large contribution to the observed background below $1 \mathrm{keV}$. This would have become even more extreme had we included line emission from metals in the emissivities. The second, Figure 15, shows the fractional contribution to the background seen by an observer at $z=0$ in several bands that was produced at various epochs (in integral form). The important point to note is that most of the X-ray background (especially in the harder bands) that we see locally was produced at relatively recent $(z \leq 0.5)$ epochs. This is a consequence of many things, prominent among them the redshift factors that dilute the observable effects of emission at high redshift.

In a later paper we will return to discuss, in greater detail, a

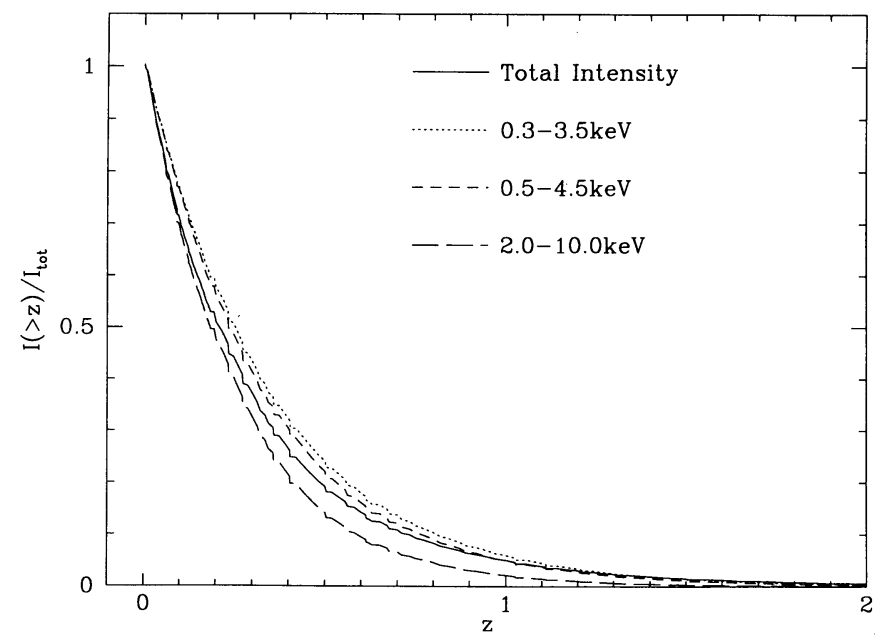

FIG. 15.-Distribution functions of four presently observed X-ray bands as a function of redshift (in integral form), indicating that most observed radiation is emitted at small redshift.

comparison between extant observations of cluster X-ray sources and the theoretical simulations.

We are happy to acknowledge support from grants NAGW2448, AST 91-08103 and ASC 93-18185. It is a pleasure to acknowledge NSCA for allowing us to use their Convex-3880 supercomputer. Discussions with Marc Davis, Patrick Henry, Mike Norman, and Simon White and an anonymous referee are gratefully acknowledged.
Babul, A., \& Katz, N. 1993, ApJ, 406, 251

Bahcall, N. A. 1993, in Astrophysical Quantities (4th ed.), in preparation Bardeen, J. M., Bond, J. R., Kaiser, M., \& Szalay, A. S. 1986, ApJ, 304, 15

Blanchard, A., Wachter, K., Evrard, A. E., \& Silk, J. 1992, ApJ, 391, 1

Bond, J. R. 1990, in The Cosmic Microwave Background: 25 Years Later, ed N. Mandolesi \& N. Vittorio (Dordrecht: Kluwer), 150

Bond, J. R., \& Myers, S. T. 1991a, in Primordial Nucleosynthesis and Evolution of Early Universe Physics, ed. K. Sato \& J. Audouze (Dordrecht: Kluwer), 305

. 1991b, in Trends in Astroparticle Physics, ed. D. Cline (Singapore: World Scientific), 24

Bryan, G. L., Cen, R. Y., Norman, M. L., Ostriker, J. P., \& Stone, J. 1994a, ApJ, in press

Bryan, G. L., Norman, M. L., Stone, J. M., Cen, R. Y., \& Ostriker, J. P. 1994b, Comput. Phys. Comm., submitted

Cavaliere, A., Menci, N., \& Setti, G. 1991, A\&A, 245, 21

Cen, R. Y. 1992, ApJS, 78, 341

Cen, R. Y., Jameson, A., Liu, F., \& Ostriker, J. P. 1990, ApJ, 362, L41

Cen, R. Y., \& Ostriker, J. P. 1992, ApJ, 393, 22 1993, ApJ, 417, 404

Cole, S., \& Kaiser, N. 1989, MNRAS, 237, 1127

Colella, P., \& Woodward, P. R. 1984, J. Comput. Phys, 54, 174

Davis, M., Efstathiou, G., Frenk, C., \& White, S. D. M. 1992, Nature, 356, 489

Efstathiou, G., Bond, J. R., \& White, S. D. M. 1992, MNRAS, 258, 1 P

Evrard, A. E. 1990, in Clusters of Galaxies, ed. W. R. Oegerle, M. Fichtel, \&

L. Danly (Cambridge: Cambridge Univ. Press), 287

Evrard, A. E., \& Henry, J. P. 1991, ApJ, 383, 95

\section{REFERENCES}

Evrard, A. E., Summers, F., \& Davis, M. 1994, ApJ, 422, 11

Frenk, C. S., White, S. D. M., Efstathiou, G., \& Davis, M. 1990, ApJ, 351, 10

Gursky, H., Kellogg, E., Murray, S., Leony, C., Tananbaum, H., \& Giacconi, R. 1971, ApJ, 167, L81

Harten, A. 1983, J. Comput. Phys., 49, 357

Henry, J. P. 1992, in Clusters and Superclusters of Galaxies, ed. A. C. Fabian (Dordrecht: Kluwer), 311

Henry, J. P., \& Arnaud, K. A. 1991, ApJ, 372, 400

Jameson, A. 1989, Science, 245, 361

Jones, W., \& Forman, C. 1992, in Clusters and Superclusters of Galaxies, ed. A. C. Fabian (Dordrecht: Kluwer), 49

Kaiser, N. 1986, MNRAS, 222, 323

Markevitch, M., Blumenthal, G. R., Forman, W., Jones, C., \& Sunyaev, R. A. 1991, ApJ, 378, L33

Ryu, D., Ostriker, J. P., Kang, H., \& Cen, R. Y. 1993, ApJ, 414, 1

Shapiro, P. R., \& Struck-Marcell, P. 1985, ApJS, 57, 205

Smoot, G. F., et al. 1992, ApJ, 396, L1

Spitzer, L., Jr. 1978, Physical Processes in the Interstellar Medium (New York: Wiley)

Walker, T. P., Steigman, G., Schramm, D. N., Olive. K. A., \& Kang, H. S. 1991, 376,51

White, S. D. M. 1991, in Clusters and Superclusters of Galaxies, ed. A. C. Fabian (Dordrecht: Kluwer), 17

Wu, X., Hamilton, T., Helfand, D. J., \& Wang, Q. 1991, ApJ, 379, 564

Yahil, A., \& Ostriker, J. P. 1973, ApJ, 185, 787

Zel'dovich, Ya. 1970, A\&A, 5, 84

Zwicky, F. 1933, Helvetica Phys. Acta, 6, 110 